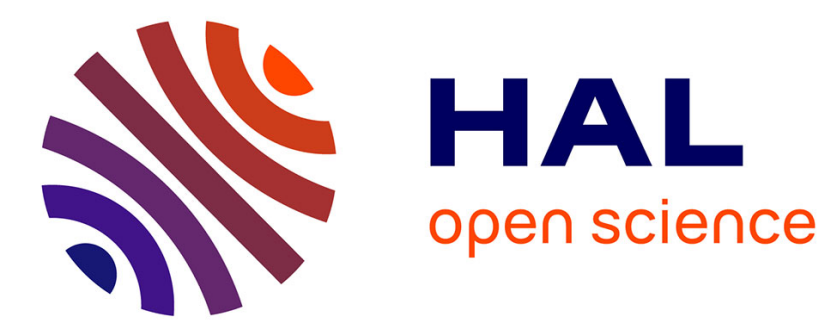

\title{
Lyapunov exponents for surface group representations.
}

Bertrand Deroin, Romain Dujardin

\section{To cite this version:}

Bertrand Deroin, Romain Dujardin. Lyapunov exponents for surface group representations.. Communications in Mathematical Physics, 2015, 340 (2), pp.433-469. hal-01068574

\section{HAL Id: hal-01068574 https://hal.science/hal-01068574}

Submitted on 26 Sep 2014

HAL is a multi-disciplinary open access archive for the deposit and dissemination of scientific research documents, whether they are published or not. The documents may come from teaching and research institutions in France or abroad, or from public or private research centers.
L'archive ouverte pluridisciplinaire HAL, est destinée au dépôt et à la diffusion de documents scientifiques de niveau recherche, publiés ou non, émanant des établissements d'enseignement et de recherche français ou étrangers, des laboratoires publics ou privés. 


\title{
LYAPUNOV EXPONENTS FOR SURFACE GROUPS REPRESENTATIONS.
}

\author{
BERTRAND DEROIN AND ROMAIN DUJARDIN
}

\begin{abstract}
Let $\left(\rho_{\lambda}\right)_{\lambda \in \Lambda}$ be a holomorphic family of representations of a surface group $\pi_{1}(S)$ into $\operatorname{PSL}(2, \mathbb{C})$, where $S$ is a topological (possibly punctured) surface with negative Euler characteristic. Given a structure of Riemann surface of finite type on $S$ we construct a bifurcation current on the parameter space $\Lambda$, that is a $(1,1)$ positive closed current attached to the bifurcations of the family. It is defined as the $d d^{c}$ of the Lyapunov exponent of the representation with respect to the Brownian motion on the Riemann surface $S$, endowed with its Poincaré metric. We show that this bifurcation current describes the asymptotic distribution of various codimension 1 phenomena in $\Lambda$. For instance, the random hypersurfaces of $\Lambda$ defined by the condition that a random closed geodesic on $S$ is mapped under $\rho_{\lambda}$ to a parabolic element or the identity are asymptotically equidistributed with respect to the bifurcation current. The proofs are based on our previous work [DD1], and on a careful control of a discretization procedure of the Brownian motion.
\end{abstract}

\section{INTRODUCTION}

Let $G$ be a finitely generated group and $\left(\rho_{\lambda}\right)_{\lambda \in \Lambda}$ be a holomorphic family of representations of $G$ into the Möbius group $\operatorname{PSL}(2, \mathbb{C})$. Viewing these representations as dynamical systems on $\mathbb{P}^{1}$, we can divide the parameter space $\Lambda$ into a stability locus, where the action of $G$ is locally quasi-conformally structurally stable, and its complement, the bifurcation locus.

In a previous article [DD1], under natural assumptions on the family $\left(\rho_{\lambda}\right)_{\lambda \in \Lambda}$, we showed how to design bifurcation currents on $\Lambda$. These are positive closed currents aiming at an understanding of the measurable and complex analytic structure of the bifurcation locus. This construction relies on probabilistic ideas, let us briefly recall some of its key steps. We start by choosing a probability measure $\mu$ on $G$, which gives rise to a random walk on $G$. An example is the nearest neighbor random walk on the Cayley graph associated to some symmetric set of generators. Fix any matrix norm $\|\cdot\|$ on $\operatorname{PSL}(2, \mathbb{C})$. Then the theory of random products of matrices implies that for any non-elementary representation $\rho$, for almost every sample path $\left(g_{n} \cdots g_{1}\right)_{n \geq 1}$ of the random walk, $\frac{1}{n} \log \left\|\rho\left(g_{n} \cdots g_{1}\right)\right\|$ converges to the Lyapunov exponent $\chi_{\mu}(\rho)$. It is easily seen that the Lyapunov exponent function $\lambda \mapsto \chi_{\mu}\left(\rho_{\lambda}\right)$ is plurisubharmonic (psh) on $\Lambda$, and we define the bifurcation current by the formula $T_{\mathrm{bif}, \mu}=d d_{\lambda}^{c} \chi_{\mu}\left(\rho_{\lambda}\right)$.

It turns out that the support of $T_{\mathrm{bif}, \mu}$ is precisely equal to the bifurcation locus. Also, $T_{\mathrm{bif}, \mu}$ describes the asymptotic distribution of certain dynamically defined sequences of subvarieties in $\Lambda$. Indeed, for $t \in \mathbb{C}$, and $g \in G$, let $Z(g, t)$ be the hypersurface in $\Lambda$ defined by

$$
Z(g, t)=\left\{\lambda \in \Lambda, \operatorname{tr}^{2}\left(\rho_{\lambda}(g)\right)=t\right\}
$$

(tr stands for the trace). The emblematic value is $t=4$, corresponding to those parameters where $\rho_{\lambda}(g)$ becomes accidentally parabolic or equal to the identity, which are known to be

Date: August 2, 2013.

B.D.'s research was partially supported by ANR-08-JCJC-0130-01, ANR-09-BLAN-0116. 
dense in the bifurcation locus. Then the following equidistribution statement holds: for almost every sample path of the random walk, the sequence of divisors $\frac{1}{2 n} Z\left(g_{n} \cdots g_{1}, t\right)$ converges to $T_{\mathrm{bif}, \mu}$ when $n \rightarrow \infty$.

We note that analogous results were previously known in the context of holomorphic families of rational mappings on $\mathbb{P}^{1}$. An essential motivation in [DD1] was actually to fill the corresponding line in the famous Sullivan dictionary between rational iteration and Kleinian group theory. The reader is referred to [Duj] for an overview on these issues.

In this paper we specialize to the case where $G=\pi_{1}(S)$ is the fundamental group of an orientable surface of genus $g$ with $n$ punctures, such that $\chi(S)=2-2 g-n<0$. As before we consider a holomorphic family $\left(\rho_{\lambda}\right)_{\lambda \in \Lambda}$ of non-elementary representations of $G$ into $\operatorname{PSL}(2, \mathbb{C})$ (we can equally deal with representations modulo conjugacy) sending simple loops about the punctures to parabolic transformations. Such representations will be referred to as parabolic. For the sake of this introduction, let us assume without further notice that these representations are faithful at generic parameters and not conjugate to each other in $\operatorname{PSL}(2, \mathbb{C})$. Our main purpose is to construct a bifurcation current on $\Lambda$ associated to the choice of a conformal structure on $S$. For this, let $X$ be a structure of Riemann surface of finite type on $S$ (that is, $X$ is biholomorphic to a compact Riemann surface minus $n$ points) and endow $X$ with its unique hyperbolic metric of curvature -1 . Recall that it is of finite volume. This data in turn gives birth to a wealth of mathematical objects:

- a dynamical system: the geodesic flow on the unit tangent bundle $T^{1} X$;

- a stochastic process: Brownian motion on $X$;

- an isomorphism between $G$ and a lattice $\Gamma$ in $\operatorname{PSL}(2, \mathbb{R})$ induced by the uniformization of $X$

We will demonstrate that our bifurcation current fits naturally with all these structures. Let us already point out that as in the previous case, this bifurcation current will be defined by taking the $d d^{c}$ of a certain Lyapunov exponent function.

A family of representations of $G$ (modulo conjugacy) naturally associated to the Riemann surface structure is obtained by considering the holonomies of holomorphic projective structures on $X$. The parameter space for this family of representations is biholomorphic to $\mathbb{C}^{3 g-3+n}$ and is the ambient space for the so-called Bers embedding of Teichmüller space. As such it plays an important role in Teichmüller theory (see Dumas [Dum] for a nice introduction to this topic). Notice that the much studied cases $(g=0, n=4)$ and $(g=1, n=1)$, corresponding to one-dimensional Teichmüller spaces, are associated to punctured surface groups. This is a motivation for allowing for non-compact surfaces, which, as we will see, are a source of technical difficulty.

We see that the space of projective structures carries a natural bifurcation current (resp. Lyapunov exponent function). The Sullivan dictionary predicts that the structure of this space should resemble that of the space of polynomials in holomorphic dynamics. In a companion paper [DD2] we introduce an invariant, the degree of a projective structure, and show that it is closely related to the Lyapunov exponent of its holonomy. This enables us to unveil a new analogy between spaces of projective structures and spaces of polynomials.

Let us discuss more precisely the contents of the present paper. As before, the definition of the bifurcation current depends on the construction of a Lyapunov exponent function on $\Lambda$, that we now describe. Mark a point $\star$ in $X$, which will serve as a base point for the fundamental group, and equipp $X$ with its Poincaré metric. For a Brownian path $\omega:[0, \infty) \rightarrow$ 
$X$ starting at $\omega(0)=\star$, we do the following construction: for $t>0$, we append $\left.\omega\right|_{[0, t]}$ with a shortest path returning to $\star$, to get a closed loop $\omega_{t}$, hence an element $\left[\omega_{t}\right] \in \pi_{1}(X, \star)-$ we disregard any possible ambiguity in the choice of the returning path.

We then have the following result

Proposition A. Let $X$ be a hyperbolic Riemann surface of finite type and $\rho: G \rightarrow \operatorname{PSL}(2, \mathbb{C})$ be a non-elementary parabolic representation. Then there exists a positive real number $\chi_{\operatorname{Brown}}(\rho)$ such that for almost every Brownian path $\omega$ starting at $\star, \frac{1}{t} \log \left\|\rho\left(\left[\omega_{t}\right]\right)\right\|$ converges to $\chi_{\mathrm{Brown}}(\rho)$ when $t \rightarrow \infty$.

In any holomorphic family $\left(\rho_{\lambda}\right)_{\lambda \in \Lambda}$ of non-elementary representations of $G$, we thus obtain a Lyapunov exponent function $\lambda \mapsto \chi_{\text {Brown }}\left(\rho_{\lambda}\right)$, and we define the bifurcation current associated to the Riemann surface structure $X$ as $T_{\text {bif }}=d d_{\lambda}^{c}\left(\chi_{\text {Brown }}\left(\rho_{\lambda}\right)\right)$. From now on we will refer to it as the natural bifurcation current. We observe that this current depends only (up to a multiplicative constant) on the conformal structure on $X$, and not on the conformal metric on $X$ which was chosen to generate the Brownian motion (in the case where $X$ has punctures some extra care is needed about the behaviour of the metric near the cusps). Indeed, by conformal invariance of the Brownian motion in dimension 2, the Lyapunov exponent function associated to any other choice of conformal metric will be a constant multiple of $\chi_{\text {Brown }}$ (see Remark 2.8 for details).

A similar procedure yields a Lyapunov exponent $\chi_{\text {geodesic }}=\chi_{\text {Brown }}$ by using a generic geodesic starting at $\star$ instead of a Brownian path (with our normalization, the Brownian motion in the hyperbolic plane escapes at unit speed). In particular $T_{\text {bif }}=d d^{c} \chi_{\text {geodesic }}$. A statement analogous to Proposition A in this context was already contained in the work of Bonatti, Gomez-Mont and Viana [BGM] (at least when $X$ is compact).

Yet another way to define the Lyapunov exponent is to use the isomorphism between $G$ and a lattice $\Gamma \leq \operatorname{PSL}(2, \mathbb{R})$ provided by uniformization. Let $\mathbb{H}$ be the hyperbolic plane. For a sequence $r_{n}$ increasing sufficiently fast to infinity (e.g. $r_{n}=n^{\alpha}$ will do), we pick at random an element $\gamma_{n} \in \Gamma \cap B_{\mathbb{H}}\left(0, r_{n}\right)$, relative to the counting measure. Then almost surely, $\frac{1}{2 r_{n}} \log \left\|\rho\left(\gamma_{n}\right)\right\|$ converges to $\chi_{\operatorname{Brown}}(\rho)$.

As in [DD1], the first main result asserts that $T_{\mathrm{bif}}$ is indeed a bifurcation current.

Theorem B. Let $X$ be a hyperbolic Riemann surface of finite type and $\left(\rho_{\lambda}\right)_{\lambda \in \Lambda}$ be a holomorphic family of non-elementary parabolic representations of $G=\pi_{1}(X, \star)$. Then the support of the natural bifurcation current $T_{\mathrm{bif}}$ is equal to the bifurcation locus.

The strategy of the proof is to rely on the corresponding support theorem in [DD1]. For this, we use a discretization procedure for the Brownian motion, which was devised by Furstenberg [F2] and then refined by Lyons and Sullivan [LS], among others. In some sense, it allows to project the Wiener measure (based, say, at $\star$ ), to a probability measure $\mu$ on $G$. We then show that the natural bifurcation current is equal to $T_{\mathrm{bif}, \mu}$, up to a multiplicative constant, from which the result follows. The point is that the support theorem in [DD1] requires an exponential moment condition on $\mu$. When $X$ is compact, this fact is apparently part of the folklore. We show that when $X$ is merely of finite volume and $\rho$ is a parabolic representation, $\mu$ still satisfies the following slightly modified moment property

$$
\int_{G} \exp \left(\beta d_{\mathbb{H}}(0, \rho(g)(0))\right) d \mu(g)<\infty \text { for some } \beta>0,
$$


that is enough to apply the results of [DD1] (see Proposition 2.16). The proof is rather technical, and occupies most of $\S 2$. We believe that it could find applications beyond this work.

The second main theme of the paper is the equidistribution of random hypersurfaces of the form $Z\left(g_{n}, t\right)$ towards the bifurcation current. Given the Riemann surface structure $X$, there are at least two natural choices for the group elements $g_{n}$ :

- A first possibility is to pick randomly an element $\gamma_{n}$ in $\Gamma \cap B_{\mathbb{H}}\left(0, r_{n}\right)$ (according to the counting measure), and let $r_{n} \rightarrow \infty$.

- A second choice is to choose at random a closed geodesic $\gamma_{n}$ of length at most $r_{n}$ on $X$. This geodesic defines a conjugacy class in $G$, so, abusing notation slightly, we can define $\operatorname{tr}^{2}(\rho([\gamma]))$ and it makes sense to consider $Z\left(\gamma_{n}, t\right)=\left\{\lambda \in \Lambda, \operatorname{tr}^{2}\left(\left[\gamma_{n}\right]\right)=t\right\}$.

In both cases, we show that the expected equidistribution holds. For concreteness, let us state precisely the equidistribution theorem for random closed geodesics (see Theorem 3.1 below for the other case). The notion of a random closed geodesic will be made precise in §3.3. Several natural models are possible, the theorem applies to all of them.

Theorem C. Let $X$ be a hyperbolic Riemann surface of finite type and $\left(\rho_{\lambda}\right)_{\lambda \in \Lambda}$ be a holomorphic family of non-elementary parabolic representations of $G=\pi_{1}(X, \star)$ into $\operatorname{PSL}(2, \mathbb{C})$. Fix $t \in \mathbb{C}$. Let $\left(r_{n}\right)$ be a sequence such that for every $c>0$, the series $\sum_{n>0} e^{-c r_{n}}$ converges. Let $\left(\gamma_{n}\right)$ be a sequence of independent random closed geodesics on $X$ of length at most $r_{n}$. Then almost surely,

$$
\frac{1}{2 \text { length }\left(\gamma_{n}\right)}\left[Z\left(\gamma_{n}, t\right)\right] \underset{n \rightarrow \infty}{\longrightarrow} T_{\text {bif }}
$$

Notice that this result does not follow from the equidistribution results in [DD1] since the random elements $\gamma_{n}$ are not obtained from a random walk on $G$. To prove the theorem, we consider the sequence of psh potentials

$$
u\left(\gamma_{n}, \lambda\right)=\frac{1}{2 \text { length }\left(\gamma_{n}\right)} \log \left|\operatorname{tr}^{2}\left(\rho_{\lambda}\left(\gamma_{n}\right)\right)-t\right| .
$$

We need to show that this sequence of psh functions a.s. converges to $\chi_{\mathrm{Brown}}\left(\rho_{\lambda}\right)$ in $L_{\mathrm{loc}}^{1}(\Lambda)$.

The main step is to study this convergence for a fixed representation.

Theorem D. Let $X$ be a hyperbolic Riemann surface of finite type and $\rho: G \rightarrow \operatorname{PSL}(2, \mathbb{C})$ be a non-elementary parabolic representation. Let $\left(r_{n}\right)$ and $\left(\gamma_{n}\right)$ be as in Theorem $C$. Then almost surely

$$
\frac{1}{2 \text { length }\left(\gamma_{n}\right)} \log \left|\operatorname{tr}^{2}\left(\rho\left(\gamma_{n}\right)\right)\right| \underset{n \rightarrow \infty}{\longrightarrow} \chi_{\operatorname{Brown}}(\rho) .
$$

Notice that when $\rho$ is the uniformizing representation (i.e. $\rho=$ id when $G$ is identified to $\left.\Gamma), \log \mid \operatorname{tr}^{2}\left(\left[\gamma_{n}\right]\right)\right) \mid$ is nothing but the length of $\gamma_{n}$ (up to an error of order $O\left(e^{-\operatorname{length}\left(\gamma_{n}\right)}\right)$ ). This theorem thus describes how this quantity evolves as $\rho$ is deformed in the space of representations.

By the Borel-Cantelli lemma, to prove Theorem D we need an estimate on the number of geodesics of length at most $r$ such that $\left|\frac{1}{2 \operatorname{length}(\gamma)} \log \right| \operatorname{tr}^{2}(\rho(\gamma))\left|-\chi_{\text {Brown }}(\rho)\right| \geq \varepsilon$. This counting is based on large deviation estimates for the traces of random products of matrices, together with a precise understanding of the Furstenberg-Lyons-Sullivan discretization procedure. Observe that the exponential moment property of the discretization measure is also crucial here. 
Finally, Theorem $\mathrm{C}$ is a rather straightforward consequence of Theorem D, by using some standard compactness properties of psh functions.

The outline of the paper is as follows. In $\S 1$ after a few facts on hyperbolic geometry and $\operatorname{PSL}(2, \mathbb{C})$ representations, we recall some necessary results from [DD1]. We also discuss the basic properties of Brownian motion on hyperbolic surfaces. In $\S 2$, we define the natural Lyapunov exponent and prove Theorem B. For further reference as well as for completeness, we include a detailed treatment of the discretization procedure. The equidistribution theorems are established in $\S 3$. We conclude the paper with a few open questions.

The results of this paper were announced in a preliminary form in [DD1, §5.1] and [Duj, $\S 4.5]$.

\section{Preliminaries}

Throughout the paper we use the following notation: if $u$ and $v$ are two real valued functions, we write $u \asymp v(\operatorname{resp} u \lesssim v)$ if there exists a constant $C>0$ such that $\frac{1}{C} u \leq v \leq C u$ (resp. $u \leq C v$ ). We also use the letter $C$ for a generic positive "constant", which may change from line to line, independently of a varying parameter which should be clear from the context.

1.1. Plane hyperbolic geometry. To fix notation, we summarize a few basic facts from hyperbolic geometry. See Beardon [Bea] for a systematic account. We let $\mathbb{H}^{2}$ (often simply $\mathbb{H}$ ) be the hyperbolic plane (resp. $\mathbb{H}^{3}$ be the hyperbolic 3 -space) of curvature -1 . The hyperbolic distance is denoted by $d_{\mathbb{H}}$. The hyperbolic area in $\mathbb{H}^{2}$ is simply denoted by by Area $(\cdot)$, and we recall that the area of a ball of radius $r$ is $4 \pi \sinh ^{2} \frac{r}{2}$. As usual, we may switch from the Poincaré disk (resp. ball) to the upper half plane (resp. space) model depending on the particular situation. The group of orientation preserving isometries of $\mathbb{H}^{2}$ (resp. $\mathbb{H}^{3}$ ) will be viewed as $\operatorname{PSL}(2, \mathbb{R})$ (resp. $\operatorname{PSL}(2, \mathbb{C})$ ). The $\mathrm{SO}(2)$ (resp. $S U(2)$ ) invariant point will be denoted by 0 . We let $\|\cdot\|$ be the operator norm associated to the standard Hermitian metric on $\mathbb{C}^{2}$; recall that $\|A\|$ is the spectral radius of $A^{*} A$. The norm of an element $\gamma \in \operatorname{PSL}(2, \mathbb{C})$ is by definition $\|A\|$, where $A$ is any matrix representative of $\gamma$ in $\operatorname{SL}(2, \mathbb{C})$. We also sometimes use $\|\cdot\|_{2}$ defined by $\|\gamma\|_{2}:=\left(|a|^{2}+|b|^{2}+|c|^{2}+|d|^{2}\right)^{1 / 2}$, where $A=\left(\begin{array}{ll}a & b \\ c & d\end{array}\right)$. Likewise, if $\gamma \in \operatorname{PSL}(2, \mathbb{C}), \operatorname{tr}^{2}(\gamma)$ and $|\operatorname{tr}(\gamma)|$ are well-defined quantities. Norm and displacement are related by the identity

$$
\|\gamma\|_{2}^{2}=2 \cosh \left(d_{\mathbb{H}}(0, \gamma 0)\right)
$$

in particular $2 \log \|\gamma\| \sim d_{\mathbb{H}}(0, \gamma 0)$ as $\|\gamma\| \rightarrow \infty$. If $\gamma$ is a hyperbolic (resp. loxodromic) element then we denote its translation length by $\ell(\gamma)$, which satisfies

$$
\cosh \left(\frac{\ell(\gamma)}{2}\right)=\frac{1}{2}|\operatorname{tr}(\gamma)| .
$$

1.2. Spaces of representations of $\pi_{1}(S)$ into $\operatorname{PSL}(2, \mathbb{C})$. A good source for the material in this section is [Kap]. Let $S$ be a (topological) surface of finite topological type, that is, homeomorphic to a compact surface of genus $g$ with a finite number $n$ of punctures. Throughout the paper we always assume that $\chi(S)=2-2 g-n<0$. If $S$ is endowed with a complex structure, the corresponding Riemann surface will be denoted by $X$. Fix a basepoint $\star \in S$ and put 
$G=\pi_{1}(S, \star)$. The space of representations of $\operatorname{Hom}(G, \operatorname{PSL}(2, \mathbb{C}))$ is an affine complex algebraic variety (recall that $\operatorname{PSL}(2, \mathbb{C})$ is affine because $\operatorname{PSL}(2, \mathbb{C}) \simeq \operatorname{SO}(3, \mathbb{C})$ ). A general holomorphic family of representations is simply a holomorphic mapping $\Lambda \rightarrow \operatorname{Hom}(G, \operatorname{PSL}(2, \mathbb{C}))$. The group $\operatorname{PSL}(2, \mathbb{C})$ acts algebraically by conjugation on $\operatorname{Hom}(G, \operatorname{PSL}(2, \mathbb{C}))$, and the algebraic quotient $\operatorname{Hom}(G, \operatorname{PSL}(2, \mathbb{C})) / / \operatorname{PSL}(2, \mathbb{C})$ is the character variety $\mathcal{X}(G)$. Coordinates on $\mathcal{X}(G)$ are given by functions of the form $\rho \mapsto \operatorname{tr}^{2}(\rho(\gamma))$. We also let $\mathcal{X}^{N E}(G) \subset \mathcal{X}(G)$ be the subset corresponding to non-elementary representations.

When $S$ is not compact we actually rather work with the relative character variety $\mathcal{X}_{\mathrm{par}}(G)$, which is defined as follows. Let $\left(h_{i}\right)_{i=1 \ldots n}$ in $G$ be classes of closed loops turning once around the punctures. We define the set of parabolic representations by

$$
\operatorname{Hom}_{\text {par }}(G, \operatorname{PSL}(2, \mathbb{C}))=\left\{\rho \in \operatorname{Hom}(G, \operatorname{PSL}(2, \mathbb{C})), \forall i, \rho\left(h_{i}\right) \text { is parabolic }\right\} .
$$

The algebraic quotient $\mathcal{X}_{\text {par }}(G)=\operatorname{Hom}_{\text {par }}(G, \operatorname{PSL}(2, \mathbb{C})) / / \operatorname{PSL}(2, \mathbb{C})$ is the relative character variety. It can be shown that $\mathcal{X}_{\mathrm{par}}(G)$ is of complex dimension $6 g-6+2 n$, and is smooth at non-elementary representations.

1.3. Bifurcation currents. Here we gather some material from [DD1]. See also [Duj] for a general account on bifurcation currents in one-dimensional dynamics, and [Bea, Kap] for basics on Kleinian (and more generally Möbius) groups.

Let $G$ be as above, $\Lambda$ be a connected complex manifold and $\rho=\left(\rho_{\lambda}\right)_{\lambda \in \Lambda}$ be a holomorphic family of representations of $G$ into $\operatorname{PSL}(2, \mathbb{C})$. We usually work under the following assumptions:

(R1) the family is non-trivial, in the sense that there exists $\lambda_{1}, \lambda_{2}$ such that the representations $\rho_{\lambda_{i}}, i=1,2$ are not conjugate in $\operatorname{PSL}(2, \mathbb{C})$

(R2) there exists $\lambda_{0} \in \Lambda$ such that $\rho_{\lambda_{0}}$ is faithful;

(R3) for every $\lambda \in \Lambda, \rho_{\lambda}$ is non-elementary.

It may sometimes be useful to replace (R3) by the following weaker statement

(R3') there exists $\lambda_{0} \in \Lambda$ such that $\rho_{\lambda_{0}}$ is non-elementary.

In that case the subset $E \subset \Lambda$ of elementary representations is a proper real-analytic subset. According to Sullivan [Su2] there exists a partition $\Lambda=$ Bif $\cup$ Stab of $\Lambda$ into an open locus Stab of stable representations (in the sense of dynamical systems) and a closed bifurcation locus Bif. By definition, a representation $\rho$ is stable if nearby representations in $\Lambda$ are quasiconformally conjugate to $\rho$.

Fix a probability measure $\mu$ on $G$, whose support generates $G$ as a semi-group. We will assume the following locally uniform exponential moment condition:

(M) $\forall \lambda_{0} \in \Lambda, \exists N\left(\lambda_{0}\right)$ neighbd of $\lambda_{0}$, and $\sigma>0$ s.t. $\forall \lambda \in N\left(\lambda_{0}\right), \int_{G}\left\|\rho_{\lambda}(g)\right\|^{\sigma} d \mu(g)<\infty$,

where $\|\cdot\|$ is any matrix norm on $\operatorname{PSL}(2, \mathbb{C})$. Under such a moment condition one can define a Lyapunov exponent function on $\Lambda$ by the formula

$$
\chi_{\mu}(\lambda)=\lim _{n \rightarrow \infty} \frac{1}{n} \int \log \left\|\rho_{\lambda}(g)\right\| d \mu^{n}(g),
$$

and the associated bifurcation current $T_{\mathrm{bif}, \mu}=d d^{c} \chi_{\mu}$-we deliberately emphasize the dependence on $\mu$ here.

The following result was established in [DD1, Thm. 3.13]. 
Theorem 1.1. Let $(G, \rho, \mu)$ be a holomorphic family of representations of $G$, satisfying (R1, $\left.R 2, R 3^{\prime}\right)$, endowed with a measure $\mu$, generating $G$ as a semi-group, and satisfying $(M)$. Then $\operatorname{Supp}\left(T_{\mathrm{bif}, \mu}\right)$ is equal to the bifurcation locus.

A comment is in order here: the theorem was stated in [DD1] under the more restrictive moment condition

$$
\text { there exists } s>0 \text { s.t. } \int_{G} \exp (s \text { length }(g)) d \mu(g)<\infty,
$$

where the length is relative to some finite system of generators of $G$. But, as explained in the beginning of [DD1, §3.3], it still holds under (M).

It is important to observe that these notions also make sense on (resp. relative) character varieties. Indeed let $\Lambda$ be a piece of complex submanifold of the set of characters of non elementary representations of $G$ into $\operatorname{PSL}(2, \mathbb{C}), \Lambda \subset \mathcal{X}^{N E}(G)\left(\right.$ resp. $\left.\Lambda \subset \mathcal{X}_{\text {par }}^{N E}(G)\right)$. Then it can be lifted to a holomorphic family of representations, transverse to the foliation by conjugacy classes, so we can define a stability (resp. bifurcation) locus on $\Lambda$, which does not depend on the choice of lifting. Likewise, the validity of the moment condition (M), as well as the value of the Lyapunov exponent, are independent of the lift. Hence there is a well-defined bifurcation current on $\Lambda$, and Theorem 1.1 holds.

1.4. Brownian motion. More details on the material in this paragraph can be found in [CC, Gri, FL] Let $X$ be a complete hyperbolic Riemann surface, endowed with its hyperbolic metric. Brownian motion on $X$ is defined as the Markov process with transition probability $P_{t}(x, \cdot)=p_{t}(x, y) d y$, where the volume element $d y$ is relative to the hyperbolic metric, and the heat kernel $p_{t}$ satisfies

$$
\frac{\partial}{\partial t} p_{t}(x, \cdot)=\Delta p_{t}(x, \cdot) \text { and } \lim _{t \rightarrow 0} p_{t}(x, \cdot) d x=\delta_{x}
$$

(It is also common to work with $\frac{1}{2} \Delta$. We choose this normalization to stay in accordance with the usual convention in foliation theory (see $[\mathrm{CC}]$ )). This process is a diffusion with continuous sample paths so it gives rise for every $x \in X$ to a probability measure $W_{x}$ (the Wiener measure) on the space $\Omega_{x}$ of continuous paths $\omega:[0, \infty[\rightarrow X$ such that $\omega(0)=x$ (itself endowed with the compact open topology). We also denote by $\Omega$ the space of continuous paths $\omega:\left[0, \infty\left[\rightarrow X\right.\right.$ and put $W=\int W_{x} d x$. We often the classical notation $\mathbb{P}_{x}$ (resp. $\mathbb{E}_{x}$ ) for the probability (resp. expectation) of some event relative to $W_{x}$.

It is useful to think of sample paths of the Brownian motion on $X \simeq \Gamma \backslash \mathbb{H}^{2}$ as projections of Brownian paths on $\mathbb{H}^{2}$. To say it differently, the Wiener measures associated to a point in $X$ and of one of its lifts to $\mathbb{H}^{2}$ are naturally identified. With our normalization, the linear drift of Brownian motion on $\mathbb{H}^{2}$ is equal to 1 , that is, for $W_{0}$-a.e. Brownian path $\omega, d_{\mathbb{H}}(0, \omega(t)) \sim t$ as $t \rightarrow \infty$ (see the appendix in [DDp] for an elementary proof).

We will require the following precise estimate for the radial density $k^{0}(t, r)$ of the law at time $t$ of Brownian motion issued from 0 (i.e. $\left.P_{t}(0, \cdot)\right)$ in $\mathbb{H}^{2}$

$$
k^{0}(t, r) \asymp\left(1+r^{-1}\right)^{-1} t^{-1}(1+r+t)^{-1}(1+r) \exp \left(-\frac{(r-t)^{2}}{4 t}\right)
$$

(see e.g. [Da, Thm 5.7.2]). 


\section{The Lyapunov exponent associated to Brownian motion}

Let $S$ be a topological surface of genus $g$ with $n$ punctures, with a marked point $\star$, and such that $\chi(S)<0$. Recall that $G=\pi_{1}(S, \star)$. In $\S 2.1$ we show that the data of a structure of Riemann surface of finite type on $S$ gives rise to a natural Lyapunov exponent function $\chi_{\text {Brown }}$ on $\operatorname{Hom}_{\text {par }}\left(G, \operatorname{PSL}(2, \mathbb{C})\right.$ ) (resp. $\mathcal{X}_{\text {par }}(G)$ ). Proving that $d d^{c} \chi_{\text {Brown }}$ is a genuine bifurcation current occupies the remainder of this section. Our main tool is a discretization procedure for Brownian motion, which will be discussed thoroughly in $\S 2.2$. From this point, the main issue is to obtain estimates for a probabilistic stopping time, associated to the discretization. The case where $X$ is compact is substantially simpler, and will be discussed in $\S 2.3$. In $\S 2.4$, we deal with the general case. The proof of Theorem 2.7 is given in $\S 2.5$. In $\S 2.6$, we re-interpret these results from the geodesic point of view, from which Proposition A follows.

2.1. Definition. Let $X$ be a structure of Riemann surface of finite type on $S$ (i.e. a neighborhood of each puncture is biholomorphic to $\left.\mathbb{D}^{*}\right)$. The Euler characteristic assumption implies that $X$ is hyperbolic, and we endow $X$ with its hyperbolic metric of curvature -1 . Let $(\widetilde{X}, \star)$ be the universal cover of $(X, \star)$. We fix an isomorphism between $\widetilde{X}$ and $\mathbb{H}^{2}$ mapping $\star$ to 0 . Then the deck transformation group $G$ becomes naturally isomorphic to a lattice $\Gamma \leq \operatorname{PSL}(2, \mathbb{R})$. We let $\rho_{\text {can }}$ be the (Fuchsian) uniformizing representation of $G$ such that $\Gamma=\rho_{\text {can }}(G)$, and in the sequel we conveniently identify $G$ and $\Gamma$ when needed.

Fix a representation $\rho \in \operatorname{Hom}(G, \operatorname{PSL}(2, \mathbb{C}))$. If $\gamma$ is any loop on $X$ based at $\star$, and $[\gamma] \in G$ denotes its class (from now on we drop the square brackets), we may consider the quantity $\log \|\rho(\gamma)\|$.

We are now ready to define the natural Lyapunov exponent of a parabolic representation of $G$, associated to the complex structure on $S$. Justifying the validity of this definition will occupy the remainder of this subsection.

Definition-Proposition 2.1. Let $X$ be a hyperbolic Riemann surface of finite type and $\rho: \pi_{1}(X, \star) \rightarrow \operatorname{PSL}(2, \mathbb{C})$ be a parabolic representation

Define a family of loops as follows: for $t>0$, consider a Brownian path $\omega$ issued from $\star$, and concatenate $\left.\omega\right|_{[0, t]}$ with a shortest geodesic joining $\omega(t)$ and $\star$, thus obtaining a closed loop $\widetilde{\omega}_{t}$.

Then for $W_{\star}$ a.e. $\omega$ the limit

$$
\chi_{\text {Brown }}(\rho)=\lim _{t \rightarrow \infty} \frac{1}{t} \log \left\|\rho\left(\widetilde{\omega}_{t}\right)\right\|
$$

exists and does not depend on $\omega$. This number is by definition the Lyapunov exponent of $\rho$, associated to the complex structure $X$.

Notice that there is a little ambiguity in the definition for there might be several shortest paths joining $\omega(t)$ and $\star$. As it will be clear from the proof, this does not affect the existence nor the value of the limit in (5). It will also follow from the proof that $\chi_{\text {Brown }}$ does not depend on $\star$. Since with our normalization, the escape rate of the Brownian motion in $\mathbb{H}^{2}$ is 1 , we deduce from $(1)$ that $\chi_{\text {Brown }}\left(\rho_{\text {can }}\right)=\frac{1}{2}$.

To prove the existence of $\chi_{\text {Brown }}$ and study its properties, we will need to consider the holonomy of non-closed paths on $X$. For this, we work on the suspension of $\rho$. For any representation $\rho \in \operatorname{Hom}(G, \operatorname{PSL}(2, \mathbb{C}))$, the suspension of $\rho$ is by definition the quotient of $\mathbb{H}^{2} \times \mathbb{P}^{1}$ under the diagonal action of $G$. To be specific, $M_{\rho}=\mathbb{H}^{2} \times \mathbb{P}^{1} / \sim$ where the equivalence relation is defined by $(p, z) \sim\left(p^{\prime}, z^{\prime}\right)$ if there exists $g \in G$ such that $\left(p^{\prime}, z^{\prime}\right)=(g(p), \rho(g)(z))$. 
The natural projection $\pi: M_{\rho} \rightarrow X$ makes $M_{\rho}$ a flat $\mathbb{P}^{1}$-bundle over $X$, and the horizontal foliation on $\mathbb{H}^{2} \times \mathbb{P}^{1}$ descends to $M_{\rho}$ as a holomorphic foliation with leaves transverse to the $\mathbb{P}^{1}$ fibers, whose monodromy representation is $\rho$.

The point is to study the growth rate of holonomy between vertical fibers in $M_{\rho}$ along generic Brownian paths in $X$, measured with respect to some smooth family of spherical metrics on the fibers. When $X$ is compact the choice of the metrics on fibers is harmless. On the other hand in the non-compact case this requires some care, so let us dwell a little bit on this point.

By a spherical metric, we mean a conformal metric on $\mathbb{P}^{1}$ of curvature 1. Equivalently, it is the Fubini-Study metric associated to some Hermitian structure on $\mathbb{C}^{2}$. Fix a smooth family of spherical metrics on the fibers of $M_{\rho}$. If $\omega$ is any continuous path on $X$, we denote by $h_{\rho}(\omega)$ its holonomy $h_{\rho}(\omega): \pi^{-1}(\gamma(0)) \rightarrow \pi^{-1}(\gamma(1))$. We define $\left\|h_{\rho}(\omega)\right\|$ as $\sup _{x \in \pi^{-1}(\gamma(0))}\left\|d_{x} h_{\rho}(\omega)\right\|$, where the norm is relative to the choice of conformal metrics on the fibers.

We say that the family of spherical metrics is normalized if the induced metric on $\pi^{-1}(\star)$ is the standard Fubini-Study metric. This means that the natural map $\{0\} \times \mathbb{P}^{1} \rightarrow \pi^{-1}(\star)$ induced by the definition of $M_{\rho}$ as a quotient, is an isometry when $\mathbb{P}^{1}$ is given the standard Fubini-Study metric. This condition is meant to ensure that for closed loops, the definition of $\left\|h_{\rho}(\omega)\right\|$ coincides with the natural one.

By definition a family of spherical metrics on $M_{\rho}$ is Lipschitz if there exists a constant $C_{\rho}>0$ such that for every smooth path $\omega$ on $X$, it holds that

$$
\log \left\|h_{\rho}(\omega)\right\| \leq C_{\rho} \operatorname{length}(\omega),
$$

where the length is relative to the hyperbolic metric on $X$.

When $X$ is compact any smooth family of metrics is of course Lipschitz. In the non compact case we have the following result.

Proposition 2.2. If $\rho$ is a parabolic representation of $G$, then there exists a smooth normalized Lipschitz family of spherical metrics on the fibers of $M_{\rho}$. Furthermore the constant $C_{\rho}$ in (6) can be chosen to be locally uniformly bounded on $\operatorname{Hom}_{\mathrm{par}}(G, \operatorname{PSL}(2, \mathbb{C}))$.

Notice that the result is false for a general representation. Indeed consider a path of length $L$ turning about $e^{L}$ times around a cusp of $X$. Let $g \in G$ representing a simple loop aroung that cusp. Then if $\rho(g)$ is loxodromic, we see that (6) will be violated. On the other hand it can be shown that the proposition (as well as all results in this section) remains true as soon as every such $\rho(g)$ has eigenvalues only on the unit circle.

Proof. The first observation is that the set of spherical metrics on $\mathbb{P}^{1}$ is parameterized by $\mathbb{H}^{3}$. This follows from the fact that such a metric is the visual metric $\theta(p)$ from a point $p \in \mathbb{H}^{3}$ on the sphere at infinity $\mathbb{P}^{1}$. Another way to see it is to observe that a spherical metric is defined by a Möbius map from $\mathbb{P}^{1}$ (equipped with the standard Fubini-Study metric) up to precomposition by an isometry, and $\operatorname{PSL}(2, \mathbb{C}) / \operatorname{PSU}(2) \simeq \mathbb{H}^{3}$.

We denote by $d s_{p}$ the metric associated to $p \in \mathbb{H}^{3}$. We claim that if $p, q \in \mathbb{H}^{3}$, we have that

$$
\log \left\|\frac{d s_{p}}{d s_{q}}\right\|_{\infty}=d_{\mathbb{H}}(p, q)+O(1) .
$$

This follows from an elementary computation. Assume first that $q=0$, so that $d s_{q}^{2}=d s_{0}^{2}=$ $\frac{|d z|^{2}}{\left(1+|z|^{2}\right)^{2}}$ is the usual spherical metric. Let $p \in \mathbb{H}^{3}, p=A^{-1}(0)$ for some $A \in \operatorname{PSL}(2, \mathbb{C})$, so 
that $d s_{p}=A^{*} d s_{0}$. Putting $A=\left(\begin{array}{ll}a & b \\ c & d\end{array}\right)$, we infer that

$$
d s_{p}=\frac{|d z|}{|a z+b|^{2}+|c z+d|^{2}},
$$

therefore $\left\|\frac{d s_{p}}{d s_{0}}\right\|_{\infty}=\left\|A^{-1}\right\|^{2}=\|A\|^{2}$, and finally

$$
\log \left\|\frac{d s_{p}}{d s_{0}}\right\|_{\infty}=2 \log \|A\|=d_{\mathbb{H}}(0, p)+O(1) .
$$

To handle the general case, pick an isometry $h$ of $\mathbb{H}^{3}$ bringing $q$ back to 0 , and observe that since $\frac{d s_{p}}{d s_{q}}$ is a function on $\mathbb{P}^{1}$,

$$
\left\|\frac{h^{*} d s_{p}}{h^{*} d s_{q}}\right\|_{\infty}=\left\|h^{*} \frac{d s_{p}}{d s_{q}}\right\|_{\infty}=\left\|\frac{d s_{p}}{d s_{q}}\right\|_{\infty} .
$$

A smooth family of spherical metrics on a trivial line bundle $D \times \mathbb{P}^{1}$ is now simply the data of a smooth map $f: D \rightarrow \mathbb{H}^{3}$. A smooth family of spherical metrics on the fibers of $\mathbb{H}^{2} \times \mathbb{P}^{1}$ descends to a family of spherical metrics on $M_{\rho}$ if and only if the associated map $f$ is $\rho$-equivariant, i.e. for every $g \in G, f \circ \rho_{\text {can }}(g)=\rho(g) \circ f$. Furthermore the family is Lipschitz iff $f$ is, and the constant $C_{\rho}$ is controlled by the Lipschitz constant of $f$. This follows from (7), since the holonomy of the horizontal foliation on $\mathbb{H}^{2} \times \mathbb{P}^{1}$ is the identity.

To prove the proposition it is thus enough to show that there exists a smooth Lipschitz $\rho$-equivariant map $f: \mathbb{H}^{2} \rightarrow \mathbb{H}^{3}$. The family of spherical metrics will be normalized provided $f(0)=0$. To construct such an $f$, recall that since $X$ is of finite type, there exists a finite number of open disjoint subsets $C_{1}, \ldots, C_{r}$ of $X$ such that $X \backslash \bigcup_{i} C_{i}$ is compact, and each $C_{i}$ is isometric to the quotient of $\left\{z \in \mathbb{H}^{2} \mid \Im z>\rho_{i}\right\}$. by the translation $z \mapsto z+1$ for some positive $\rho_{i}$. For every $i=1, \ldots, r$, we pick some lift $\widetilde{C_{i}} \subset \mathbb{H}^{2}$ of $C_{i}$, and we first define $f$ on the closure of $\widetilde{C}_{i}$. Denote by $g_{i} \in G$ a generator of the stabilizer of $\widetilde{C}_{i}$ in $G$. By assumption, $\rho\left(g_{i}\right)$ is parabolic, hence conjugate to $g_{i}$. Thus, there exists an isometric immersion $f: \widetilde{\widetilde{C}_{i}} \rightarrow \mathbb{H}^{3}$ such that $f \circ g_{i}=\rho\left(g_{i}\right) \circ f$ (we take the map in $\operatorname{PSL}(2, \mathbb{C})$ which conjugates $g_{i}$ to $\left.\rho\left(g_{i}\right)\right)$. We extend the map $f$ by $\rho$-equivariance to a map defined on $\bigcup_{i} G \overline{\widetilde{C}_{i}}$. Then, because $\mathbb{H}^{3}$ is contractible, a classical topological argument shows that the map $f$ can be extended as a smooth $\rho$-equivariant map from $\mathbb{H}^{2}$ to $\mathbb{H}^{3}$ such that $f(0)=0$. We claim that this map is Lipschitz. Indeed, $\|D f\|$ is $G$-invariant (the norm of $D f$ is measured using hyperbolic metrics on source and target), thus descends to a function on $X$. But since $f$ is an isometric embedding on $\widetilde{C_{i}}$, we have $\|D f\|=1$ on the cusps $C_{i}$ 's. Hence $\|D f\|$ is bounded, since $X \backslash \cup_{i} C_{i}$ is compact, and $f$ is indeed our desired smooth Lipschitz $\rho$-equivariant map. Finally, it is clear that the Lipschitz constant of $f$ can be chosen to be locally uniformly bounded in $\operatorname{Hom}_{\text {par }}(G, \operatorname{PSL}(2, \mathbb{C}))$, so the same is true of the constant $C_{\rho}$.

The following consequence of the previous proposition will be useful.

Corollary 2.3. For every parabolic representation $\rho$ there exists a constant $\beta_{\rho}$ depending only on the constant $C_{\rho}$ of $(6)$ such that $\|\rho(g)\| \leq\left\|\rho_{\text {can }}(g)\right\|^{\beta_{\rho}}$. 
Proof. Indeed for $g \in G$, let $\sigma_{g}$ be the geodesic segment $\left[0, \rho_{\text {can }}(g) 0\right]$ in $\mathbb{H}^{2}$. Then in $M_{\rho}$, the holonomy of the projection of $\sigma_{g}$ in $X$ is $\rho(g)$. By (1), (6) and the normalization of the family of spherical metrics we infer that

$$
\log \left\|h_{\rho}\left(\sigma_{g}\right)\right\|=\log \|\rho(g)\| \leq C_{\rho} \operatorname{length}\left(\sigma_{g}\right) \leq C_{\rho} \operatorname{argcosh} \frac{\left\|\rho_{\text {can }}(g)\right\|_{2}^{2}}{2} \leq C_{\rho} \log \left(2\left\|\rho_{\text {can }}(g)\right\|^{2}\right) .
$$

Here we use the inequalities $\|\gamma\|_{2}^{2} \leq 2\|\gamma\|^{2}$ and $\operatorname{argcosh} x \leq \log 2 x$. Hence we get that $\|\rho(g)\| \leq\left(2\left\|\rho_{\text {can }}(g)\right\|\right)^{2 C_{\rho}}$. To conclude the proof, just note that since $\rho_{\text {can }}(g)$ contains no elliptic transformations, there exists $c>0$ such that $\left\|\rho_{\text {can }}(g)\right\|>1+c$, so there exists $\alpha$ such that $2 \leq\left\|\rho_{\text {can }}(g)\right\|^{\alpha}$. The result follows.

We have the following analogue of Definition 2.1 in this context.

Proposition 2.4. Let $X$ be a hyperbolic Riemann surface of finite type, $\rho: \pi_{1}(X) \rightarrow$ $\operatorname{PSL}(2, \mathbb{C})$ be a parabolic representation, and $\|$.$\| a smooth normalized Lipschitz family of$ spherical metrics on $M_{\rho}$. With notation as above, for $W$-a.e. path $\omega$, the limit

$$
\chi(\rho ; \omega)=\lim _{t \rightarrow \infty} \frac{1}{t} \log \left\|h_{\rho}\left(\left.\omega\right|_{[0, t]}\right)\right\|
$$

exists and does not depend on $\omega$ nor on the the choice of $\|\cdot\|$.

Proof. Let $\sigma$ be the shift semi-group acting on $\Omega$ by $\sigma_{s}: \omega(\cdot) \mapsto \omega(\cdot+s)$. Recall that $W=$ $\int W_{x} d x$. Since the Riemannian measure is invariant under heat flow, $W$ is invariant under the time shift $\sigma_{s}: \omega(\cdot) \mapsto \omega(\cdot+s)$. The family of functionals $H_{t}(\omega): \omega \mapsto \log \left\|h_{\rho}\left(\left.\omega\right|_{[0, t]}\right)\right\|$ defines a sub-additive cocycle. In order to apply Kingman's subadditive ergodic theorem, we need to check that this cocycle is $W$-integrable, that is, that $\int \log \left\|d h_{\rho}\left(\left.\omega\right|_{[0, t]}\right)\right\| d W(\omega)<\infty$ for some (hence every) $t>0$. For this we use the Lipschitz property of the family of spherical metrics. Let $\widehat{X}$ be a Dirichlet fundamental domain containing 0 for the action of $\Gamma$ on $\mathbb{H}^{2}$. For a Brownian path $\omega$, let $\widetilde{\omega}$ be its lift to $\mathbb{H}^{2}$ starting from the lift of $\omega(0)$ lying in $\widetilde{X}$. By (6) we have that

$$
\log \left\|h_{\rho}\left(\omega_{\mid[0, t]}\right)\right\| \lesssim d_{\mathbb{H}}(\widetilde{\omega}(0), \widetilde{\omega}(t))+d_{\mathbb{H}}(0, \widetilde{\omega}(0)) .
$$

Thus

$$
\int \log \left\|d h_{\rho}\left(\left.\omega\right|_{[0, t]}\right)\right\| d W(\omega) \lesssim \int_{\widetilde{X}} \mathbb{E}_{x}\left(d_{\mathbb{H}}(\widetilde{\omega}(0), \widetilde{\omega}(t))\right) d x
$$

which is finite thanks to the (super)exponential decay of the heat kernel on hyperbolic plane (cf. (4)).

The following result finally justifies the validity of Definition 2.1.

Proposition 2.5. Let $X, \rho$, and \|\| be as in Proposition 2.4. Then for $W_{\star}$ a.e. Brownian path $\omega$, we have that

$$
\lim _{t \rightarrow \infty} \frac{1}{t} \log \left\|\rho\left(\widetilde{\omega}_{t}\right)\right\|=\lim _{t \rightarrow \infty} \frac{1}{t} \log \left\|h_{\rho}\left(\left.\omega\right|_{[0, t]}\right)\right\|=\chi_{\operatorname{Brown}}(\rho) .
$$

Furthermore the limit in (8) is also equal to $\chi_{\mathrm{Brown}}(\rho)$.

Proof. Let us first show that for $W_{\star}$ a.e. $\omega, \lim _{t \rightarrow \infty} \frac{1}{t} \log \left\|h_{\rho}\left(\left.\omega\right|_{[0, t]}\right)\right\|$ exists and has the same value as the one in (8). Indeed the diffusion of the Dirac mass $\delta_{\star}$ at time 1 is absolutely continuous with respect to volume measure on $X$. Now we simply write $h_{\rho}\left(\left.\omega\right|_{[0, t]}\right)=$ $h_{\rho}\left(\left.\omega\right|_{[1, t]}\right) \circ h_{\rho}\left(\left.\omega\right|_{[0,1]}\right)$ and by the Markov property of Brownian motion together with Proposition 2.4, we get that for $W_{\star}$ a.e. $\omega, \frac{1}{t} \log \left\|\left.\omega\right|_{[1, t]}\right\|$ converges to the limit in (8). 
To finish the proof, we show that for $W_{\star}$ a.e. $\omega$,

$$
\frac{1}{t}\left|\log \left\|\rho\left(\widetilde{\omega}_{t}\right)\right\|-\log \left\|h_{\rho}\left(\left.\omega\right|_{[0, t]}\right)\right\|\right| \underset{t \rightarrow \infty}{\longrightarrow} 0 .
$$

If $X$ is compact this is obvious since $d_{X}(\omega(t), \star)$ is uniformly bounded. In the general case, by the Lipschitz property we have to show that for $W_{\star}$ a.e. $\omega, d_{X}(\omega(t), \star)=o(t)$. A way to see this is to use the following two facts: first, a theorem of Sullivan [Su1] asserts that for almost every $v$ in the unit tangent space $S_{\star} X$ to $\star$, the unit speed geodesic ray $\gamma(t)$ issued from $\star$ pointing in the direction $v$ satisfies $d_{X}(\star, \gamma(t))=O(\log t)$. The other result we use is the classical fact that Brownian paths on hyperbolic space are shadowed by geodesic rays (see e.g. [An]). More precisely, if $\omega$ is a Brownian path starting from $0 \in \mathbb{H}^{2}$, then $\lim _{t \rightarrow \infty} \omega(t)=\omega_{\infty}$ exists in $\partial \mathbb{H}^{2}$, and if $\gamma_{x, \omega_{\infty}}$ denotes the unit speed geodesic ray joining $x$ and $\omega_{\infty}$, then for $W_{0}$ a.e. $\omega, d_{\mathbb{H}}\left(\omega(t), \gamma_{x, \omega_{\infty}}(t)=o(t)\right.$ (this is actually $O(\log t)$ by a theorem of Ancona [An]). Note that the distribution of $\omega_{\infty}$ is absolutely continuous on the circle at infinity (with density given by the Poisson kernel). Therefore on $X$, for $W_{\star}$ a.e. $\omega$, there exists a unit tangent vector such that $d_{X}\left(\omega(t), \gamma_{\star, v}(t)\right)=o(t)$. Since the distribution of the tangent vector $v$ is induced by that of $\omega_{\infty}$, it is absolutely continuous with respect to Lebesgue measure on $S_{\star} X$. So we can combine this estimate with Sullivan's, and conclude that $d_{X}(\omega(t), \star)=o(t)$ a.s., which implies (9).

For further reference, we now make the easy observation that the natural Lyapunov exponent is insensitive to finite coverings.

Proposition 2.6. Let $X$ be a hyperbolic Riemann surface of finite type, and $\pi:\left(Y, y_{0}\right) \rightarrow$ $(X, \star)$ be a finite covering. Any parabolic representation $\rho: \pi_{1}(X, \star) \rightarrow \operatorname{PSL}(2, \mathbb{C})$ induces by restriction a parabolic representation $\rho_{Y}$ of $\pi_{1}\left(Y, y_{0}\right)$. Then, denoting by $\chi_{\operatorname{Brown}}\left(\rho_{Y}, Y\right)$ the Lyapunov exponent of $\rho_{Y}$ associated to the Riemann surface structure on $Y$, we have that $\chi_{\text {Brown }}(\rho, X)=\chi_{\text {Brown }}\left(\rho_{Y}, Y\right)$.

Proof. This is just a matter of following step by step the proof of the existence of $\chi_{\operatorname{Brown}}(\rho, X)$. Indeed, we observe that the suspension $Y_{\rho_{Y}}$ is naturally a covering of $M_{\rho}$, and the lift of a Lipschitz metric $\|\cdot\|$ on the fibers of $M_{\rho}$ is a Lipschitz metric on the fibers of $Y_{\rho_{Y}}$. As already observed, the Wiener spaces $W_{\star}$ on $X$ and $W_{y_{0}}$ are naturally identified to $W_{0}$ in $\mathbb{H}^{2}$. Now by construction, if $\omega$ is a Brownian path issued from $y_{0}$ in $Y$, we have that $\left\|h_{\rho_{Y}}\left(\left.\omega\right|_{[0, t]}\right)\right\|=$ $\left\|h_{\rho}\left(\left.\pi(\omega)\right|_{[0, t]}\right)\right\|$. The result follows.

Let now $\left(\rho_{\lambda}\right)_{\lambda \in \Lambda}$ be a holomorphic family of parabolic representations of $G$. Thanks to Definition 2.1 we can define a Lyapunov exponent function $\chi_{\text {Brown }}$ on $\Lambda$, and we put $T_{\text {bif }}=$ $d d^{c} \chi_{\text {Brown. }}$. We will refer to it as the natural bifurcation current of the family. The following result asserts that $T_{\text {bif }}$ is indeed a bifurcation current.

Theorem 2.7. Let $X$ be a hyperbolic Riemann surface of finite type, and $\left(\rho_{\lambda}\right)_{\lambda \in \Lambda}$ be a holomorphic family of representations of $G=\pi_{1}(X)$, satisfying (R1, R2, R3'). Then $\chi_{\text {Brown }}$ is a nonnegative psh function on $\Lambda$, and the positive closed current $T_{\mathrm{bif}}=d d^{c} \chi_{\mathrm{Brown}}$ satisfies $\operatorname{Supp}\left(T_{\text {bif }}\right)=$ Bif.

If furthermore (R3) holds then $\chi_{\text {Brown }}$ is positive and locally Hölder continuous.

The proof consists in exhibiting a measure $\mu$ on $G$ satisfying $(M)$ and such that $T_{\text {bif }, \mu}=T_{\text {bif }}$ (up to some multiplicative constant). It then suffices to apply Theorem 1.1. It will be carried out in the next subsections. 
Remark 2.8. We have constructed the natural bifurcation current by using the hyperbolic metric on $X$. Let us observe that when $X$ is compact any other conformal metric would essentially yield the same object. Indeed, choosing a different conformal metric on $X$ only changes the "clock" of Brownian motion, that is, sample Brownian paths for the new metric are time-reparameterized Brownian paths relative to the original one. It then follows from an easy ergodicity argument that the associated Lyapunov exponent function is a constant multiple of $\chi_{\text {Brown }}$. When $X$ has punctures, the same statement holds, provided one restricts to an appropriate class of conformal metrics, for instance the ones that coincide with the Poincaré metric outside some compact set of $X$.

2.2. Discretization of the Brownian motion. We will rely on a discretization procedure for the Brownian motion which was introduced by Furstenberg [F2], and subsequently studied by several authors, in particular Lyons and Sullivan [LS] (see also Kaimanovich [Kai], Ballman-Ledrappier [BaLe], Ancona [An]). Since we will need precise moment estimates, we include a detailed treatment of the discretization, which is close (but not identical to) that of Lyons and Sullivan.

Let $\Gamma$ be a finite covolume lattice in $\operatorname{PSL}(2, \mathbb{R})$, and $X=\Gamma \backslash \mathbb{H}$. Let $0 \in \mathbb{H}$ and $R>0$ such that the balls $B(\gamma 0, R)$ are disjoint, for $\gamma \in \Gamma$. Since the Brownian motion on $X$ is recurrent [Gri], for $0<r \leq R$ the set $\partial_{r}=\bigcup_{\gamma} \partial B(\gamma 0, r)$ is recurrent, namely a.e. Brownian path starting at a point $x$ hits $\partial_{r}$ in finite time. We denote by $T_{r}$ (resp. $T_{R}$ ) the first hitting time to $\partial_{r}\left(\right.$ resp. $\left.\partial_{R}\right)$. Since $\partial_{r}\left(\right.$ resp. $\left.\partial_{R}\right)$ is closed, $T_{r}$ (resp. $\left.T_{R}\right)$ is a stopping time.

2.2.1. Sub-balayage. Recall that the balayage of a finite measure $m$ on a closed recurrent set $E$ is defined to be the image of the measure $\int W_{x} d m(x)$ under the map $\omega \mapsto \omega\left(T_{E}\right)$ where $T=T_{E}(\omega)$ is the first moment where $\omega$ hits $E$.

We denote by $B_{\rho}, S_{\rho}$ respectively the ball and sphere centered at 0 and of radius $\rho$. If $y$ is a point interior to $B_{R}$, the balayage of the Dirac mass at the point $x$ on $S_{R}$ is a probability measure absolutely continuous with respect to the spherical measure $d s$ on $S_{R}$ (conveniently normalized so that $d s$ is a probability measure), the density being given by the Poisson kernel. Hence, there is a constant $0<p<1$, such that if $x$ is chosen in the sphere $S_{r}$, its balayage on $S_{R}$ is bounded from below by $p d s$.

The following lemma will allow us to select a "good" set of paths from $S_{r}$ to $S_{R}$. For every $x \in S_{r}$, a.e. Brownian path issued from $x$ intersects $S_{R}$. The hitting time is $T_{R}(\omega)$ and we denote by $\mathrm{e}(\omega)=\omega\left(T_{R}(\omega)\right)$ the exit point. We use the standard notation $\left(\mathcal{F}_{t}\right)_{t \geq 0}$ for the Brownian filtration.

Lemma 2.9. For every $x \in S_{r}$ there exists a $\mathcal{F}_{T_{R}}$-measurable set $G_{x}$, with $\mathbb{P}\left(G_{x}\right)=p$ such that $\mathrm{e}_{*}\left(1_{G_{x}} W_{x}\right)=p d s$.

In addition, reducing $p$ slightly if necessary, $G_{x}$ can be chosen so that there exists a constant $M$ such that for every $x \in S_{r}$ and $\omega \in G_{x}, T_{R}(\omega) \leq M$.

Proof. Let us first establish the lemma without the bound $M$ on the exit time. Denote by $b_{x}(y) d s(y)$ the balayage measure of $\delta_{x}$ on $S_{R}$. Notice that $b_{x}(y)>p$ for every $y \in S_{R}$. For $0 \leq t \leq 1$ consider the piece of circle

$$
C_{t}=\left\{\frac{R+r}{2} e^{i 2 \pi \theta}, 0 \leq \theta \leq 1-t\right\}
$$

We use $C_{t}$ as a "continuously opening barrier" for Brownian paths going from $x$ to $S_{R}$. For $0 \leq t \leq 1$, let $E_{t} \subset \Omega_{x}$ be the set of paths starting at $x$ that avoid $C_{t}$ before hitting $S_{R}$. For 
$t=0$ the barrier is closed so $E_{t}=\emptyset$ while for $t=1$ it is open and $W_{x}\left(E_{t}\right)=1$; observe also that for $t<t^{\prime}, C_{t} \supset C_{t^{\prime}}$ so $E_{t} \subset E_{t^{\prime}}$. Therefore, for every $y \in S_{R}, f_{y}: t \mapsto W_{x}\left(E_{t} \mid \mathrm{e}^{-1}(y)\right)$ is a continuous increasing function with $f_{y}(0)=0$ and $f_{y}(1)=1$. The continuity of $f_{y}$ follows from the fact that the set of Brownian paths (starting from $x$ and exiting at $y$ ), intersecting $E_{t^{\prime}} \backslash E_{t}$ has small measure when $t^{\prime}-t$ is small. Let $t(y) \in(0,1)$ be the unique value of $t$ such that $f_{y}(t(y))=\frac{p}{b_{x}(y)}$. We can now define

$$
G_{x}=\left\{\omega \in \Omega_{x},\left.\omega\right|_{\left[0, T_{R}(\omega)\right]} \cap C_{t(\mathrm{e}(\omega))}=\emptyset\right\} .
$$

Clearly, this set is $\mathcal{F}_{T_{R}}$ measurable (recall that e $(\omega)=\omega\left(T_{R}(\omega)\right)$ ). Since by definition $G_{x} \cap$ $\mathrm{e}^{-1}(y)=E_{t(y)} \cap \mathrm{e}^{-1}(y)$ we infer that

$$
\begin{aligned}
\mathrm{e}_{*}\left(1_{G_{x}} W_{x}\right) & =\mathrm{e}_{*} \int W_{x}\left(G_{x} \mid \mathrm{e}^{-1}(\mathrm{e}(\omega))\right) d W_{x}(\omega)=\mathrm{e}_{*} \int W_{x}\left(E_{t(\mathrm{e}(\omega)))} \mid \mathrm{e}^{-1}(\mathrm{e}(\omega))\right) d W_{x}(\omega) \\
& =W_{x}\left(E_{t(y)} \mid \mathrm{e}^{-1}(y)\right) \mathrm{e}_{*} W_{x}=W_{x}\left(E_{t(y)} \mid \mathrm{e}^{-1}(y)\right) b_{x}(y) d s(y)=p d s,
\end{aligned}
$$

which was the desired property.

To prove the second claim, observe that for every $p_{1}<p$, if $A_{x}(M) \subset W_{x}$ denotes the set of paths such that $T_{R}(\omega) \leq M$, then for $M$ sufficiently large, $\mathrm{e}_{*}\left(1_{A_{x}\left(\tau_{1}\right)} W_{x}\right) \geq p_{1} d s$, uniformly in $x$-this is a consequence of Dini's Theorem. From this point, to get the result it suffices to repeat the above proof with $1_{A_{x}\left(\tau_{1}\right)} W_{x}$ instead of $W_{x}$.

We extend by equivariance the definition of the sets $G_{x}$ to all of $\partial_{r}$ by setting $G_{\gamma x}=\gamma G_{x}$.

2.2.2. A stopping time. For every point $x_{0} \in \mathbb{H}$ and every $\omega \in \Omega_{x_{0}}$, we define a sequence of stopping times

$$
U_{0}=T_{R}(\omega)<u_{1}=u_{1}(\omega)<U_{1}=U_{1}(\omega)<\ldots<u_{n}(\omega)<U_{n}(\omega)<\ldots
$$

as follows: $U_{0}=T_{R}(\omega)$, and by induction, $u_{n}$ is the first time after $U_{n-1}$ at which $\omega$ hits $\partial_{r}$, that is $u_{n}(\omega)=U_{n-1}(\omega)+T_{r}\left(\sigma_{U_{n-1}}(\omega)\right)$, where as before $\sigma_{t}$ denotes time shift by $t$. Likewise, $U_{n}$ is the first time after $u_{n}$ at which $\omega$ hits $\partial_{R}$, i.e. $U_{n}(\omega)=u_{n}(\omega)+T_{R}\left(\sigma_{u_{n}}(\omega)\right)$.

By the strong Markov property of Brownian motion, almost surely there exists an integer $n$ such that $\sigma_{u_{n}} \omega$ belongs to $G_{\omega\left(u_{n}\right)}$. We define $T(\omega):=U_{k}(\omega)$, where $k=k(\omega)$ is the first such integer. The measurability property of the sets $G_{x}$ ensures that $T$ is a stopping time (intuitively at time $t$ we know if we already went from $\partial_{r}$ to $\partial_{R}$ through some $G_{x}$ ). We let $x_{1}(\omega)=\omega(T(\omega))$ and $r_{1}(\omega)$ be the element of $\Gamma$ such that $x_{1} \in \partial B\left(r_{1} \cdot 0, R\right)$. The law of $x_{1}$ defines a transition probability $\Pi\left(x_{0}, \cdot\right)$. By construction it satisfies the equivariance property $\Pi\left(\gamma x_{0}, \cdot\right)=\gamma_{*} \Pi\left(x_{0}, \cdot\right)$ for $\gamma \in \Gamma$. $\Gamma^{\mathbb{N}}$.

Repeating this process independently gives rise to sequences $\left(x_{n}\right)_{n \geq 0} \in\left(\mathbb{H}^{2}\right)^{\mathbb{N}}$ and $\left(r_{n}\right)_{n \geq 1} \in$

2.2.3. The discretization measure. It follows from the construction that whatever the distribution of the point $x_{0}$ is, the distribution of the point $x_{1}$ is a convex combination of the spherical measures $d s_{\gamma}=\gamma_{*} d s$ on $\partial_{R}$. Hence, if $x_{0}$ has distribution $d s$ on $S_{R}$ (observe that $U_{0}=0$ in this case), then $x_{1}$ has distribution $\mu * d s$ for a certain measure $\mu$ on $\Gamma$.

We now define a Markov chain $\left(x_{n}\right)$ with initial distribution given by the normalized spherical measure $d s$ on $S_{R}$ and transition kernel $\Pi$. By the equivariance property of $\Pi$, the distribution of $x_{n}$ is $\mu^{n} * d s$. 
Furthermore, since the transition kernel is defined by stopping the Brownian motion, by the strong Markov property we infer that the map $\omega \mapsto\left(r_{n}(\omega)\right)$ sends the measure $\int_{S_{R}} W_{x} d s(x)$ to the path measure $\mathrm{P}$ on $\Gamma^{\mathbb{N}}$ of the right random walk on $\Gamma$ induced by $\mu$.

By definition $\mu$ is the discretization measure (notice that it is not unique).

2.3. The cocompact case. The core of the proof of Theorem 2.7 is the fact that the discretization measure satisfies the exponential moment condition (M). When $X$ is compact, this fact is well-known to the specialists (for instance it is announced without proof in [An]), but also not so easy to find in print. Let us explain this case first. Recall that $\Gamma=\rho_{\text {can }}(G)$.

Proposition 2.10. If the lattice $\Gamma$ is cocompact, then the discretization measure $\mu$ satisfies the exponential moment condition (3) with respect to word length in $G$.

Proof. By the stronger assertion of Lemma 2.9, for every $p_{1}<p$ there exists a deterministic time $\tau_{1}$ such that for every $x \in S_{r}$ there exists a $\mathcal{F}_{T_{R}}$ measurable set $G_{x}$ such that $\mathrm{e}_{*}\left(1_{G_{x}} W_{x}\right)=$ $p_{1} d s$ and moreover for every $\omega \in G_{x}^{1}, T_{R}(\omega) \leq \tau_{1}$.

On the other hand, since $X=\Gamma \backslash \mathbb{H}$ is compact, there exists $0<p_{2}<1$ and $\tau_{2}>0$ such that for every $x \in \mathbb{H}$, the probability that a Brownian path starting at $x$ hits $\partial_{r}$ in time less than $\tau_{2}$ is bounded from below by $p_{2}$.

Therefore, by the Markov property we infer that for every $x \in \mathbb{H}$ and every $t_{0}$,

$$
\mathbb{P}_{x}\left(T \geq t_{0}+\tau_{1}+\tau_{2}\right) \leq\left(1-p_{1} p_{2}\right) \cdot \mathbb{P}_{x}(T \geq t),
$$

where $T$ is the stopping time defined in $\S 2.2 .2$. From this we deduce that there exist constants $C, \alpha>0$ independent of $x$ such that

$$
\mathbb{P}_{x}(T \geq t) \leq C e^{-\alpha t}
$$

Since the discretization measure is the image of $\int_{S_{R}} W_{x} d s(x)$ under $\omega \mapsto \omega(T(\omega))$, and the distance between $r_{1}(\omega) \cdot 0$ and $\omega(T(\omega))$ is $R$, for every $\delta>0$, we have that

$$
\mu(\gamma \in \Gamma, d(\gamma 0,0) \geq \delta) \leq \int \mathbb{P}_{x}(d(\omega(T(\omega)), 0) \geq \delta-R) d s(x),
$$

where $d(\cdot, \cdot)$ denotes hyperbolic distance. Fix $K>1$ as in Lemma 2.11 below. For every $x \in S_{R}$, we have that

$$
\begin{aligned}
\mathbb{P}_{x}(d(\omega(T), 0) \geq \delta-R) & \leq \mathbb{P}_{x}(d(\omega(T), x) \geq \delta-2 R) \\
& \leq \mathbb{P}_{x}\left(\sup _{t \leq(\delta-2 R) / K} d(\omega(t), x) \geq(\delta-2 R)\right)+\mathbb{P}_{x}\left(T \geq \frac{\delta-2 R}{K}\right) .
\end{aligned}
$$

By Lemma 2.11 below the first term decreases exponentially fast when $\delta$ tends to infinity, while the second does because of (10). By the homogeneity of hyperbolic plane, these estimates are uniform for $x \in S_{R}$, and me conclude that $\mu(\gamma \in \Gamma, d(\gamma(0), 0) \geq \delta)$ decreases exponentially with $\delta$. Finally, since $\Gamma=\rho_{\text {can }}(G)$ is cocompact, $(\Gamma 0, d)$ is quasi-isometric to any Cayley graph of $G$ and we are done.

The following result is presumably well-known but we could not locate a precise reference.

Lemma 2.11. There exists a positive constant $K$ such that

$$
\mathbb{P}_{0}\left(\sup _{0 \leq s \leq t} d(0, \omega(s)) \geq K t\right)
$$

decreases exponentially with $t$. 
Proof. Let us work in the disk model of hyperbolic plane. The expression for the hyperbolic distance is $\exp (d(0, z))=\frac{1+|z|}{1-|z|}$. Since $x \mapsto \frac{1+x}{1-x}$ is convex and increasing, $z \mapsto \exp (d(0, z))$ is a subharmonic function. From this we infer that if $\omega(t)$ is the Brownian motion starting at 0 , the process defined by $(\exp (d(0, \omega(t))))_{t>0}$ is a positive submartingale.

From the asymptotic estimate for the heat kernel given in $\S 1.4$, we infer that $\mathbb{E}_{0}(\exp (d(0, \omega(t))))$ is finite for every $t$. Now we have that

$$
\begin{aligned}
\mathbb{E}_{0}(\exp (d(0, \omega(t)))) & \leq \mathbb{E}_{0}(\exp (d(0, \omega(\lceil t\rceil)))) \text { by the submartingale property } \\
& \leq \mathbb{E}_{0}\left(\exp \left(\sum_{i=0}^{\lceil t\rceil-1} d(\omega(i), \omega(i+1))\right)\right) \text { by the triangle inequality } \\
& =\mathbb{E}_{0}(\exp (d(0, \omega(1))))^{\lceil t\rceil} \text { by the Markov property } \\
& \leq \mathbb{E}_{0}(\exp (d(0, \omega(1))))^{t+1}
\end{aligned}
$$

(for the equality on the third line we take successive conditional expectations and use the homogeneity of the hyperbolic plane). The Doob martingale inequality applied to $\exp (d(0, \omega(t)))$ asserts that for every $\alpha>0$,

$$
\mathbb{P}_{0}\left(\sup _{0 \leq s \leq t} \exp d(0, \omega(s)) \geq \alpha\right) \leq \frac{1}{\alpha} \mathbb{E}_{0}(\exp (d(0, \omega(t))))
$$

Hence if $K$ is any real number larger than $\mathbb{E}_{0}(\exp (d(0, \omega(1))))$, applying inequality (12) with $\alpha=\exp (K t)$ together with (11) finishes the proof.

Remark 2.12. It is likely that the result holds for any $K>1$. A possible way to achieve this would be to refine the subdivision of the interval $[0, t]$ in (11).

2.4. The finite volume case. We will need the following estimate on the hitting time of the Brownian motion on a given subset of $X$. We thank J. Franchi for explaining it to us.

Proposition 2.13. Let $X$ be a hyperbolic Riemann surface of finite type, endowed with its Poincaré metric, and Liouville measure $d x$. Let $K \subset X$ be a compact set of positive area and define a stopping time for the Brownian motion by

$$
T_{K}(\omega)=\inf \{t>0, \omega(t) \in K\} .
$$

Then there exists a constant $\alpha>0$ such that for every $x \in X, \mathbb{E}_{x}\left(e^{\alpha T_{K}}\right)<+\infty$. Furthermore, the function $x \mapsto \mathbb{E}_{x}\left(e^{\alpha T_{K}}\right)<+\infty$ is locally bounded and in $L^{2}(X, d x)$.

Of course this uniformity is highly non-trivial for some paths go deeply inside a cusp before reaching $K$.

Proof. Let $\left(\Pi_{t}\right)_{t \geq 0}$ be the Brownian semigroup on $X$, associated to $\Delta$. The spectral theory of the Laplacian on $X$ implies that $\Pi_{t}$ is strongly contracting, specifically there exists a constant $c>0$ such that for every $f \in L^{2}(X)$ of zero mean, $\left\|\Pi_{t} f\right\|_{L^{2}} \leq e^{-c t}\|f\|_{L^{2}}$ (see e.g. [FL] for an elementary approach to the Poincaré inequality on $X$, which classically implies a positive lower bound for the first eigenvalue of the Laplacian). Therefore the family of operators defined by $Q_{T}=\frac{1}{T} \int_{0}^{T} \Pi_{t}$ converges strongly to $f \mapsto \int_{X} f=\int f(x) d x$ in $L^{2}$. More precisely there exists a function $c(T)$ with $\lim _{+\infty} c(T)=0$ such that

$$
\left\|Q_{T} f-\int_{X} f\right\|_{L^{2}} \leq c(T)\|f\|_{L^{2}} \text {. }
$$


Indeed write $f=f_{0}+\int f$ with $\int f_{0}=0$. Notice that $\left\|f_{0}\right\|_{L^{2}} \leq 2\|f\|_{L^{2}}$. Then we have that $\left\|Q_{T} f-\int f\right\|_{L^{2}}=\left\|Q_{T} f_{0}\right\|_{L^{2}}=\left\|\frac{1}{T} \int_{0}^{T_{0}} \Pi_{t} f_{0}+\frac{1}{T} \int_{T_{0}}^{T} \Pi_{t} f_{0}\right\| \leq \frac{2}{T}\|f\|_{L^{2}}+\frac{T-T_{0}}{T} e^{-c T}\|f\|_{L^{2}}$.

In this setting, a theorem by Carmona and Klein [CK, Thm 1] asserts that $x \mapsto \mathbb{E}_{x}\left(e^{\alpha T_{K}}\right)$ belongs to $L^{2}(X)$, in particular it is finite a.e. Furthermore, elaborating on [CK, Rmk 1], we see that $\mathbb{E}_{x}\left(e^{\alpha T_{K}}\right)$ is locally bounded. Indeed let $t_{0}>0$, and use the Markov property to write

$$
\begin{aligned}
& \mathbb{E}_{x}\left(e^{\alpha T_{K}}\right)=\int_{\left\{T_{k} \leq t\right\}} e^{\alpha T_{K}} d W_{x}+\int_{\left\{T_{k}>t_{0}\right\}} e^{\alpha T_{K}} d W_{x} \leq e^{\alpha t_{0}}+e^{\alpha t_{0}} \int \mathbb{E}_{y}\left(e^{\alpha T_{K}}\right) p_{t_{0}}(x, y) d y \\
& \leq e^{\alpha t_{0}}+e^{\alpha t_{0}}\left\|\mathbb{E}_{y}\left(e^{\alpha T_{K}}\right)\right\|_{L^{2}}\left\|p_{t_{0}}(x, \cdot)\right\|_{L^{2}},
\end{aligned}
$$

where $\Pi_{t}(x, y) d y$ is the transition kernel. Now, as it easily follows from the estimates in $\S 1.4$, for fixed $t_{0}$ the heat kernel $p_{t_{0}}(x, \cdot)$ is in $L^{2}$, locally uniformly with respect to $x$. The result follows.

From this proposition we get estimates on the stopping times $T_{r}$ and $T_{R}$ in $\mathbb{H}$.

Corollary 2.14. There exists uniform constants $\alpha_{0}$ and $M$ such that for every $x \in \partial_{R}$, $\mathbb{E}_{x}\left(\exp \alpha_{0} T_{r}\right) \leq M$ (resp. for every $\left.x \in \partial_{r}, \mathbb{E}_{x}\left(\exp \alpha_{0} T_{R}\right) \leq M\right)$.

Proof. To prove the corollary we work on $X$. Abusing notation we set $\partial_{r}=\pi S_{r}$, and similarly for $\partial_{R}$, where $\pi: \mathbb{H}^{2} \rightarrow X$ is the natural projection. Recall that by assumption $B(0, R)$ projects to a ball on $X$. Assume that $x \in \partial_{R}$, and fatten $\partial_{r}$ on the side opposite to $x$ to make it a closed annulus $\widetilde{\partial}_{r}$. Then the hitting times of $\partial_{r}$ and $\widetilde{\partial_{r}}$ are the same, and we conclude by Proposition 2.13. The other case is similar.

The next step is to show that the stopping time $T$ of 2.2 .2 defining the discretization admits an exponential moment.

Proposition 2.15. With notation as in §2.2, there exist constants $\alpha$ and $M$ such that for every $x \in S_{R}$,

$$
\mathbb{E}_{x}(\exp (\alpha T)) \leq M
$$

Proof. Let $x \in S_{R}$. Recall that for a.e. $\omega \in W_{x}$ we defined in $\S 2.2 .2$ the integer $k(\omega)$ as the first time at which $\omega\left(u_{n}(\omega)\right)$ enters the good set $G_{\omega\left(u_{n}\right)}$. For a constant $\alpha$ to be determined later, we decompose the integral $\int \exp (\alpha T) d W_{x}$ as a sum

$$
\sum_{K} \int_{\Omega_{x}^{k}} \exp (\alpha T) d W_{x}
$$

where $\Omega_{x}^{K}=\{\omega \mid k(\omega)=K\}$. We define a sequence of shifted paths $\omega_{l}, 0 \leq l \leq 2 K-1$ by $\omega_{0}=\omega, \omega_{1}=\sigma_{u_{1}}(\omega), \omega_{2}=\sigma_{U_{1}}(\omega)$, etc. We also define for $1 \leq l \leq 2 K-1, y_{l}(\omega)=\omega_{l}(0)$, so that for even $l$ (resp. odd $l$ ), $y_{l} \in S_{R}$ (resp. $y_{l} \in S_{r}$ ). Notice that by definition of $K$, for $l=2 j-1, j<K, \omega_{l} \notin G_{y_{l}}$ while $\omega_{2 K-1} \in G_{y_{2 K-1}}$.

Writing $T(\omega)=T_{r}\left(\omega_{0}\right)+T_{R}\left(\omega_{1}\right)+\cdots+T_{R}\left(\omega_{2 K-1}\right)$, taking successive conditional expectations with respect to the events $T_{\bullet}\left(\omega_{l}\right)=y_{l+1}($ with $\bullet=r$ or $R$ ) and applying the strong 
Markov property yields

$$
\begin{aligned}
& \int_{\Omega_{x}^{k}} \exp (\alpha T) d W_{x}=\int_{\Omega_{x}} \exp \left(\alpha T_{r}\left(\omega_{0}\right)\right) d W_{x}\left(\omega_{0}\right) \cdots \\
& \quad \ldots \int_{G_{y_{2 j-1}}^{c}} \exp \left(\alpha T_{R}\left(\omega_{2 j-1}\right)\right) d W_{y_{2 j-1}}\left(\omega_{2 j-1}\right) \int_{\Omega_{y_{2 j}}} \exp \left(\alpha T_{r}\left(\omega_{2 j}\right)\right) d W_{y_{2} j}\left(\omega_{2 j}\right) \cdots \\
& \quad \ldots \int_{G_{y_{2 K-1}}} \exp \left(\alpha T_{R}\left(\omega_{2 K-1}\right)\right) d W_{y_{2 K-1}}\left(\omega_{2 K-1}\right) .
\end{aligned}
$$

Now we claim that for every $\beta>0$ there exists $\alpha>0$ such that putting $q=1-p$ we have

(i) $\forall x \in S_{R}, \int \exp \left(\alpha T_{r}\right) d W_{x} \leq e^{\beta}$

(ii) $\forall y \in S_{r}, \int_{G_{y}^{c}} \exp \left(\alpha T_{R}\right) d W_{y} \leq q e^{\beta}$ and $\int_{G_{y}} \exp \left(\alpha T_{R}\right) d W_{y} \leq p e^{\beta}$.

From this and (13) we immediately get that

$$
\int_{\Omega_{x}^{K}} \exp (\alpha T) d W_{x} \leq e^{2 K \beta} q^{K-1} p
$$

Thus, choosing $\beta$ such that $e^{2 \beta} q<1$ and summing over $K$, we obtain that

$$
\int \exp (\alpha T) d W_{x} \leq \frac{p}{q} \sum_{K}\left(e^{2 \beta} q\right)^{K}<+\infty
$$

and the result follows.

It remains to prove our claim. We only deal with (ii), the other case being analogous. Let $0<\alpha<\alpha_{0} / 2$, where $\alpha_{0}$ is in Corollary 2.14, let $\beta^{\prime}=\beta / 2$ and set $E=G_{y}$ or $E=G_{y}^{c}$. Applying the Chebychev inequality, Corollary 2.14 implies that for every $y \in \partial_{r}$, we have

$$
W_{y}\left(T_{R} \geq t\right) \leq M \exp \left(-\alpha_{0} t\right) .
$$

We partition the set $E$ as

$$
E=\left(E \cap\left\{\alpha T_{R} \leq \beta^{\prime}\right\}\right) \cup \bigcup_{n=0}^{\infty}\left(E \cap\left\{\beta^{\prime}+\alpha n<T_{R}<\beta^{\prime}+\alpha(n+1)\right\}\right)
$$

and decompose the integral $\int_{E} \exp \left(\alpha T_{R}\right) d W_{y}$ accordingly, which yields

$$
\begin{aligned}
\int_{E} \exp \left(\alpha T_{R}\right) d W_{y} & \leq e^{\beta} W_{y}(E)+\sum_{n} \mathbb{P}_{x}\left(T_{R} \geq \frac{\beta^{\prime}}{\alpha}+n\right) e^{\beta^{\prime}+\alpha(n+1)} \\
& \leq e^{\beta^{\prime}} W_{x}(E)+C \exp \left(-\frac{\alpha_{0} \beta^{\prime}}{\alpha}\right)
\end{aligned}
$$

with $C=\frac{e^{\alpha_{0}+\beta^{\prime}} M}{1-e^{-\alpha_{0} / 2}}$. Note that $W_{x}(E)$ equals $p$ or $q$, that are both fixed positive numbers, so that if $\alpha$ is sufficiently small we have that

$$
\int_{E} \exp \left(\alpha T_{R}\right) d W_{y} \leq e^{\beta^{\prime}} W_{x}(E)+C \exp \left(-\frac{\alpha_{0} \beta^{\prime}}{\alpha}\right) \leq e^{\beta} W_{x}(E) .
$$

and claim (ii) follows. The proof is complete.

We can now show that the discretization measure satisfies the moment condition (M). 
Proposition 2.16. Let $X$ be a hyperbolic Riemann surface of finite type, $\rho$ be a parabolic representation of $G=\pi_{1}(X)$ and $\mu$ be the discretization of the Brownian motion. Then there is a constant $\alpha=\alpha_{\rho}>0$ such that

$$
\int\|\rho(g)\|^{\alpha} d \mu(g)<\infty
$$

Furthermore, the constant $\alpha_{\rho}$ can be chosen to be locally uniform in $\operatorname{Hom}_{\text {par }}(\Gamma, \operatorname{PSL}(2, \mathbb{C}))$. In this case the value of the integral in (15) is also locally uniform.

Proof. For $\rho=\rho_{\text {can }}$, given Proposition 2.15, the proof is identical to that of Proposition 2.10. However in this case we cannot transfer the moment condition in $\Gamma$ to a moment condition with respect to word length in $G$ (see Remark 2.17 below). Instead we use the Lipschitz property of the spherical metrics on the fibers of $M_{\rho}$ to obtain moment estimates for $\rho(G)$.

Let us be more precise. The proof of Proposition 2.10 shows that the exponential decay property (10) implies that

$$
\mu(\gamma \in \Gamma, d(\gamma(0), 0) \geq \delta) \leq e^{-c \delta}
$$

for some positive constant $c$. Since $\|\gamma\| \asymp \log d(\gamma 0,0)$ we infer that there exists $\alpha>0$ such that

$$
\int_{G}\left\|\rho_{\text {can }}(g)\right\|^{\alpha} d \mu(g)<\infty .
$$

Applying Corollary 2.3 thus finishes the proof.

Remark 2.17. When $X$ is not compact, the moment condition in (15) cannot be replaced by (3). Actually, $\mu$ does not even have a finite moment with respect to word length in $G$, i.e. $\int_{G}$ length $(g) d \mu(g)=\infty$. This was shown (somehow implicitly) by Guivarc'h and Le Jan in [GL]. See also [DKN, Corollary 1.22] for a different approach, as well as Gruet [Gru, Rmk p. 500] for a neat computation in the case of the modular surface.

2.5. Proof of Theorem 2.7. Let $\mu$ be the discretization measure and $\chi_{\mu}$ be the associated Lyapunov exponent. We showed in Proposition 2.16 that $\mu$ satisfies the moment condition $(\mathrm{M})$. Furthermore, it is easy to see that $\operatorname{Supp}(\mu)=G$. Indeed, recall the balayage construction of $\mu$. We start with the circle measure $d s$ on $S_{R}$ and "sub-balayage" it successively on $\partial_{R}=\Gamma \cdot S_{R}$. The first step of the process is to balayage $d s$ onto $\partial_{r}=\Gamma \cdot S_{r}$. It is clear that the image measure has positive mass on every circle $\gamma S_{r}, \gamma \in \Gamma$. Now for each circle $\gamma S_{r}$, there is a set of positive measure of good paths, that leaves some measure on $\gamma S_{R}$ (for the remaining paths, we repeat the process over again). Therefore $\mu$ puts some mass on $\{\gamma\}$.

By Theorem 1.1, $\operatorname{Supp}\left(d d^{c} \chi_{\mu}\right)=$ Bif. To prove the theorem, we show that there exists a positive constant $c$ such that $\chi_{\text {Brown }}=c \chi_{\mu}$, hence $\operatorname{Supp}\left(T_{\text {bif }}\right)=\operatorname{Supp}\left(T_{\text {bif }, \mu}\right)=$ Bif. Under (R3), the positivity and Hölder continuity properties of $\chi_{\text {Brown }}$ then follow from classical results due to Furstenberg [F1] and Le Page [Le] .

Let $T$ be the stopping time defining $\mu$, and $T_{n}$ be the sequence of times obtained by repeating the stopping process. Let $W_{S_{R}}$ be defined by $W_{S_{R}}=\int_{S_{R}} W_{x} d s(x)$. For $W_{S_{R}}$ a.e. $\omega, \omega\left(T_{n}\right) \in r_{n}(\omega) S_{R}$, with $\left(r_{n}\right) \in \Gamma^{\mathbb{N}}$ as in $\S 2.2 .2$.

Identify $\Gamma$ and $G$ through $\rho_{\text {can }}$ and consider a general parabolic representation $\rho$. Let as before $\sigma_{r_{n}}$ denote the geodesic segment joining 0 and $r_{n}(0)$. Since $d\left(r_{n}(0), \omega\left(T_{n}\right)\right)=R$ is constant we infer that

$$
\frac{1}{n}\left|\log \left\|h_{\rho}\left(\sigma_{r_{n}(\omega)}\right)\right\|-\log \left\|h_{\rho}\left(\omega\left(T_{n}\right)\right)\right\|\right|=\frac{1}{n}\left|\log \left\|\rho\left(r_{n}\right)\right\|-\log \left\|h_{\rho}\left(\omega\left(T_{n}\right)\right)\right\|\right| \longrightarrow 0 .
$$


Since the law of $r_{n}$ is $\mu^{n}$ we thus deduce that

$$
\chi_{\mu}(\rho)=\lim _{n \rightarrow \infty} \frac{1}{n} \int_{G} \log \|\rho(g)\| d \mu^{n}(g)=\lim _{n \rightarrow \infty} \frac{1}{n} \int \log \left\|h_{\rho}\left(\omega\left(T_{n}\right)\right)\right\| d W_{S_{R}}(\omega) .
$$

To conclude the proof, it remains to show that there exists a constant $\tau>0$ such that $\frac{T_{n}}{n} \rightarrow \tau$ for $W_{S_{R}}$ a.e. $\omega$. This, together with Proposition 2.5, implies that the right hand side of (16) equals $\tau \chi_{\text {Brown }}$

To prove this, we view $S_{R}$ as a subset of $X$. Let $\theta_{T}$ be the time shift by $T(\omega)$, acting on the set of Brownian paths $\omega$ issued from $S_{R}$, equipped with the measure $W_{S_{R}}$. By construction the law of $\omega(T)$ is $d s(x)$. From this fact and the strong Markov property we see that $\left(\theta_{T}\right)_{*} W_{S_{R}}=$ $W_{S_{R}}$. Furthermore there cannot be any measurable $\theta_{T}$-invariant subset, again by the strong Markov property together with the fact that for every $x \in S_{R}$, the law of $\omega(T)$, conditioned to start at $x$, is $d s(x)$. Therefore $\theta_{T}$ is $W_{S_{R}}$-ergodic

To conclude, observe that $T_{n}(\omega)=\sum_{k=0}^{n-1} T\left(\theta_{T}^{k} \omega\right)$ and recall from Proposition 2.15 that $T$ is integrable. Thus, property (ii) follows from Birkhoff's ergodic theorem. The proof is complete.

2.6. The Lyapunov exponent associated to the geodesic flow. As before let $X$ be a Riemann surface of finite type with a marked point $\star$, endowed with its hyperbolic metric. For $v \in T_{\star}^{1} X$ a unit tangent vector, we let $\gamma_{v}$ be the associated unit speed geodesic ray. These geodesics form another natural class of paths on $X$. We also denote by $\widetilde{\gamma}_{v, t}$ the loop obtained by appending to $\left.\gamma_{v}\right|_{[0, t]}$ a shortest path returning to $\star$ (as before, we disregard the possible ambiguities happening here). This point of view allows to define a Lyapunov exponent function, similarly to Definition 2.1. It turns out that it coincides with $\chi_{\text {Brown }}$ up to a multiplicative constant. Proposition A immediately follows.

Theorem 2.18. Let $X$ be a hyperbolic Riemann surface of finite type, endowed with its Poincaré metric. Let $\rho: \pi_{1}(X, \star) \rightarrow \operatorname{PSL}(2, \mathbb{C})$ be a parabolic representation, and $\|\cdot\| a$ smooth Lipschitz family of spherical metrics on the suspension $M_{\rho}$. Then for a.e. $v$ the limit

$$
\chi_{\text {geodesic }}(\rho):=\lim _{t \rightarrow \infty} \frac{1}{t} \log \left\|h_{\rho}\left(\left.\gamma_{v}\right|_{[0, t]}\right)\right\|=\lim _{t \rightarrow \infty} \frac{1}{t} \log \left\|h_{\rho}\left(\widetilde{\gamma}_{v, t}\right)\right\|
$$

exists and is equal to $\chi_{\operatorname{Brown}}(\rho)$ (in particular it does not depend on $v$ ).

We leave the reader check that the same result holds for geodesics rays issued from generic $(x, v) \in T^{1} X$ (relative to the Liouville measure), that is, $\lim _{t \rightarrow \infty} \frac{1}{t} \log \left\|h_{\rho}\left(\left.\gamma_{(x, v)}\right|_{[0, t]}\right)\right\|=$ $\chi_{\text {Brown }}(\rho)$.

Proof. The work was already done in Proposition 2.5. It follows from (6) that if $\omega_{1}$ and $\omega_{2}$ are two paths on $X$ with $\omega_{1}(0)=\omega_{2}(0)$ and such that the respective lifts $\widetilde{\omega}_{1}$ and $\widetilde{\omega}_{2}$ to $\mathbb{H}^{2}$, starting from the same point, satisfy $d_{\mathbb{H}}\left(\widetilde{\omega}_{1}(t), \widetilde{\omega}_{2}(t)\right)=o(t)$, then

$$
\lim _{t \rightarrow \infty} \frac{1}{t}\left|\log \left\|h_{\rho}\left(\omega_{1}(t)\right)\right\|-\log \left\|h_{\rho}\left(\omega_{2}(t)\right)\right\|\right|=0 .
$$

As already said, generic Brownian paths on $\mathbb{H}^{2}$ are shadowed by geodesic rays. That is, if $\omega$ is a Brownian path starting from $0 \in \mathbb{H}^{2}$, then $\lim _{t \rightarrow \infty} \omega(t)=\omega_{\infty}$ exists in $\partial \mathbb{H}^{2}$, and $d_{\mathbb{H}}\left(\omega(t), \gamma_{0, \omega_{\infty}}(t)\right)=o(t)$, where if $\gamma_{0, \omega_{\infty}}$ is the unit speed geodesic ray joining 0 and $\omega_{\infty}$.

To prove the theorem, we use this reasoning in the reverse direction. Taking endpoints of semi-infinite geodesics gives the natural "visibility" identification between $T_{0}^{1} X$ and $\partial \mathbb{H}^{2}$, sending circle measure on $T_{0}^{1} X$ to the harmonic measure $\nu_{0}$ associated to 0 on $\partial \mathbb{H}^{2}$. For 
$v \in T_{\star}^{1} X$, let $\omega_{\infty}(v)$ be the associated endpoint. For a.e. Brownian path $\omega$ conditioned to converge to $\omega_{\infty}(v)$ we have that

$$
\lim _{t \rightarrow \infty} \frac{1}{t}\left|\log \left\|h_{\rho}\left(\gamma_{v}(t)\right)\right\|-\log \left\|h_{\rho}(\omega(t))\right\|\right|=0 .
$$

Now by Proposition 2.4, for $W_{\star}$ a.e. $\omega$ we have that $\lim \frac{1}{t} \log \left\|h_{\rho}(\omega(t))\right\|=\chi_{\text {Brown }}$. Hence $\lim \frac{1}{t} \log \left\|h_{\rho}\left(\gamma_{v}(t)\right)\right\|=\chi_{\text {Brown }}(\rho)$. To deal with the loops $\widetilde{\gamma}_{v, t}$, as in Proposition 2.5 we use Sullivan's result that almost surely, $d_{X}\left(\gamma_{v}(t), \star\right)=O(\log t)$. The proof is complete.

Remark 2.19. In [BGM], Bonatti, Gomez-Mont and Viana study $\operatorname{PSL}(n, \mathbb{C})$ cocycles over hyperbolic and symbolic dynamical systems, with applications to suspensions of fundamental groups of compact surfaces. In particular they show, that if $\rho: \pi_{1}(S) \rightarrow \operatorname{PSL}(2, \mathbb{C})$ is a nonelementary representation, then the foliated geodesic flow of $M_{\rho}$ admits non-zero Lyapunov exponents (i.e. $\chi_{\text {geodesic }}(\rho)>0$ ). We see that the above methods (discretization of Brownian motion, etc.) give a new approach to this result, as it was recently observed by Alvarez [Al] (for compact $S$ ). The novelty here is that we are able to deal with parabolic representations on Riemann surfaces of finite type as well.

\section{Equidistribution in PARAmeter SPACE}

Let as before $X$ be a structure of Riemann surface of finite type on $S$. Identifying $(\widetilde{X}, \star)$ with $(\mathbb{H}, 0)$ makes $G=\pi_{1}(S, \star)$ isomorphic to a lattice $\Gamma \leq \operatorname{PSL}(2, \mathbb{R})$. From now on, we consistently identify $G$ and $\Gamma$. We endow $\Gamma$ with the metric structure induced by $\mathbb{H}$ on $\Gamma \cdot 0$, that is for $\gamma \in \Gamma$ we put $d(\gamma)=d_{\mathbb{H}}(0, \gamma 0)$; observe that $d(\gamma) \sim 2 \log \|\gamma\|$. Notice that when $X$ is not compact, this distance is not quasi-isometric to word-length in $G$. We also put $\left.B_{\Gamma}(r)=\{\gamma \in \Gamma, d(\gamma)<r)\right\}$.

This metric structure gives rise to a natural notion of a random diverging sequence in $\Gamma$ : fix a sequence $\left(r_{n}\right)_{n \geq 1}$ increasing to infinity and independently choose $\gamma_{n} \in B_{\Gamma}\left(r_{n}\right)$ relative to the counting measure. For technical reasons we will assume that the series $\sum_{n>0} e^{-c r_{n}}$ converges for every $c>0$. The resulting random sequences $\left(\gamma_{n}\right)$ will be qualified as admissible.

Let now $\Lambda$ be a holomorphic family of parabolic representations of $G$ into $\operatorname{PSL}(2, \mathbb{C})$ (resp. a holomorphic family of representations modulo conjugacy) satisfying (R1, R2, R3). For $t \in \mathbb{C}$, we consider the subvariety in $\Lambda$ defined by

$$
Z(\gamma, t)=\left\{\lambda \in \Lambda, \operatorname{tr}^{2}\left(\rho_{\lambda}(\gamma)\right)=t\right\}
$$

(which is viewed as a variety, that is, possibly with multiplicity), and the associated integration current $[Z(\gamma, t)]$. By convention if $Z(\gamma, t)=\Lambda$, we put $[Z(\gamma, t)]=0$.

Our purpose in this section is to establish the following theorem.

Theorem 3.1. Let $\Gamma$ be a torsion-free lattice in $\operatorname{PSL}(2, \mathbb{R})$, and $\left(\rho_{\lambda}\right)_{\lambda \in \Lambda}$ be a holomorphic family of parabolic representations of $\Gamma$ into $\operatorname{PSL}(2, \mathbb{C})$ satisfying $(R 1, R 2, R 3)$. Let $T_{\mathrm{Bif}}=$ $d d^{c} \chi_{\text {Brown }}$ be the natural bifurcation current, associated to Brownian motion on $X=\Gamma \backslash \mathbb{H}$. If $\left(\gamma_{n}\right)$ is an admissible random sequence in $\Gamma$, then almost surely the following convergence holds:

$$
\frac{1}{2 d\left(\gamma_{n}\right)}\left[Z\left(\gamma_{n}, t\right)\right] \underset{n \rightarrow \infty}{\longrightarrow} T_{\text {bif }}
$$

As already said in the introduction, this result cannot be deduced from the equidistribution results in [DD1]. The main step of the proof, achieved in $\S 3.1$, is to show that if $\rho \in \operatorname{Hom}_{\mathrm{par}}(\Gamma, \operatorname{PSL}(2, \mathbb{C}))$ is a fixed representation, then the exponential growth rate of 
$\operatorname{tr}^{2}\left(\rho\left(\gamma_{n}\right)\right)$ is a.s. given by $\chi_{\text {Brown }}(\rho)$. This is based on the detailed understanding of the asymptotic properties of random product of matrices associated to discretized Brownian motion.

Then in $\S 3.2$ we use Fubini's theorem and subharmonicity to get the almost sure $L_{\text {loc }}^{1}$ convergence of $\lambda \mapsto \log \left|\operatorname{tr}^{2}\left(\rho_{\lambda}\left(\gamma_{n}\right)\right)-t\right|$. From this, Theorem 3.1 immediately follows. This simple argument was suggested to us by D. Chafaï. Notice that the same approach leads to a simplification of the almost sure equidistribution theorems in [DD1] as well (see Theorems $\mathrm{B}$ and 4.1 there).

In $\S 3.3$ we show that this result can be nicely interpreted in terms of the holonomies of random sequences of closed geodesics on $X$ (Theorems D and C).

3.1. Distribution of traces in $\rho(\Gamma)$. Let $\Gamma$ be a lattice in $\operatorname{PSL}(2, \mathbb{R})$. If $\Gamma$ is torsion-free and $\rho$ is a non-elementary type-preserving representation of $\Gamma$ into $\operatorname{PSL}(2, \mathbb{C})$, the results of $\S 2$ enable to define the natural Lyapunov exponent $\chi_{\operatorname{Brown}}(\rho)$. If now $\Gamma$ has torsion, by the Selberg Lemma, we pick a finite index torsion-free subgroup $\Gamma^{\prime} \leq \Gamma$, and define $\chi_{\operatorname{Brown}}(\rho)$ to be equal to $\chi_{\text {Brown }}\left(\left.\rho\right|_{\Gamma^{\prime}}\right)$. By Proposition 2.6, the value of the Lyapunov exponent does not depend on the choice of $\Gamma^{\prime}$.

Our main result in this paragraph is the following.

Theorem 3.2. Let $\Gamma$ be a lattice in $\operatorname{PSL}(2, \mathbb{R})$, and $\rho$ be a non-elementary parabolic representation of $\Gamma$. Then for every $\varepsilon>0$ there exists $c(\varepsilon)>0$ such that for large enough $r>0$,

$$
\#\left\{\gamma \in B_{\Gamma}(r),\left|\frac{1}{2 r} \log \right| \operatorname{tr}^{2}(\rho(\gamma))\left|-\chi_{\operatorname{Brown}}(\rho)\right|>\varepsilon\right\} \leq e^{(1-c(\varepsilon)) r} .
$$

The delicate point in this theorem is the following: several times so far we have used the idea that closing a long geodesic or Brownian path by some path of bounded or short length does not affect much the norm of the holonomy. There is no such result for the trace: if $\gamma$ (resp. a) is a "large" (resp. "small") element in $\Gamma, \operatorname{tr}^{2}(\rho(\gamma))$ may be very different from $\operatorname{tr}^{2}(\rho(\gamma a))$ (even for the identity representation).

The idea of the proof is to rely on the very precise understanding of generic random products $\gamma_{1} \cdots \gamma_{n}$, where the $\gamma_{i}$ are chosen according to the discretization measure $\mu$ defined in $\S 2.2$. In Proposition 3.3 we show that we can perturb these elements in $\rho(\Gamma)$ by keeping track of the traces. We then use the properties of Brownian motion to compare $\mu^{n}$ and the counting measure, so as to cover most of a large ball in $\Gamma$.

Throughout the proof $c(\varepsilon)$ stands for a quantity which may change from line to line, dependent on $\varepsilon$ (and $\rho$ ) but not on $n$.

Our first result does not depend on Brownian motion.

Proposition 3.3. Let $\Gamma$ be as above and $\rho: \Gamma \rightarrow \operatorname{PSL}(2, \mathbb{C})$ be a non-elementary representation. Fix a probability measure $\mu$ on $\Gamma$, generating $\Gamma$ as a semi-group, and satisfying the exponential moment condition $\int_{\Gamma}\|\rho(\gamma)\|^{\alpha} d \mu(\gamma)<\infty$ for some $\alpha>0$.

Then there exists a constant $c=c(\mu, \rho)>0$ such that for every $\varepsilon>0$, when $n$ is large enough

$$
\mu^{n}\left(\left\{\gamma, \exists a \in \Gamma \text { s.t. }\|a\| \leq e^{c \varepsilon n} \text { and }\left|\frac{1}{n} \log \right| \operatorname{tr} \rho(\gamma a)\left|-\chi_{\mu}\right|>\varepsilon\right\}\right) \leq \exp (-c \varepsilon n) .
$$

We note that in this statement we could replace $\Gamma$ by an abstract finitely generated group, by replacing the condition " $\|a\| \leq e^{c \varepsilon n "}$ in (17) by "length $(a) \leq c \varepsilon n$ ". 
Proof. Without the perturbative term $a$, this was proven in [DD1, Cor. A.2] (see also [Ao]). It turns out that for fixed $a$, the proof is essentially identical to that of Theorem A.1 in [DD1], which we briefly reproduce for convenience. For a sequence $\gamma \in \Gamma^{\mathbb{N}}$, we put $\ell_{n}(\gamma)=\gamma_{1} \cdots \gamma_{n}$. We recall that if $g \in \operatorname{PSL}(2, \mathbb{C})$ has large norm, there exists two balls $A(g)$ and $R(g)$ (the attracting and repelling balls respectively) on $\mathbb{P}^{1}$, of diameter about $\frac{1}{\|g\|}$, such that $g\left(R(g)^{c}\right)=$ $A(g)$. We also recall that for $g \in \operatorname{PSL}(2, \mathbb{C})$,

$$
\frac{1}{2} \log \left|\operatorname{tr}^{2}(g)-4\right|=\log \|g\|+\log \delta(g)+O(1)
$$

where $\delta(g)$ is the distance between the fixed points of $g$. Thus to control the trace from the norm it is enough to control the distance between fixed points.

Fix $\varepsilon>0$, small with respect to $\chi$. Fix $a \in \Gamma$, with $\|a\| \leq \frac{\varepsilon}{2} n$. We have to estimate the probability that for large $n \frac{1}{n} \log \operatorname{tr}^{2}\left(\rho\left(\ell_{n}(\gamma) a\right)\right) \notin\left[\chi_{\mu}-\varepsilon, \chi_{\mu}+\varepsilon\right]$. By the large deviations theorem for the distribution of the norms [BL, $\S \mathrm{V} .6]$ we infer that outside a set of exponentially small probability, $\left|\frac{1}{n} \log \left\|\rho\left(\ell_{n}(\gamma)\right)\right\|-\chi_{\mu}\right|<\frac{\varepsilon}{4}$. Since $\|a\| \leq e^{\frac{\varepsilon}{2} n}$, we get that with high probability,

$$
\left|\frac{1}{n} \log \left\|\rho\left(\ell_{n}(\gamma) a\right)\right\|-\chi_{\mu}\right|<\frac{3 \varepsilon}{4} .
$$

Therefore we need to show that the probability that $\delta\left(\rho\left(\ell_{n}(\gamma) a\right)\right)<e^{-\frac{\varepsilon}{4} n}$ decreases like $e^{-c(\varepsilon) n}$.

For this, let $m=\lfloor(1-\varepsilon) n\rfloor$. We split $\ell_{n}(\gamma) a$ as $\ell_{n}(\gamma) a=\left(\gamma_{n} \cdots \gamma_{m+1}\right)\left(\gamma_{m} \cdots \gamma_{1} a\right)$. By the large deviations theorem for the norms, and the facts that $\|a\| \leq e^{\frac{\varepsilon}{2} n}$ and $m=\lfloor(1-\varepsilon) n\rfloor$, we get that

$$
\mathbb{P}\left(\left|\frac{1}{m} \log \left\|\rho\left(\ell_{m}(\gamma) a\right)\right\|-\chi_{\mu}\right| \geq \varepsilon\right)+\mathbb{P}\left(\log \left\|\rho\left(\gamma_{n} \ldots \gamma_{m+1}\right)\right\| \geq 2 \chi_{\mu}(n-m)\right) \leq e^{-c(\varepsilon) n} .
$$

By independence, we may assume that $\gamma_{1}, \ldots, \gamma_{m}$ are fixed and satisfy $\left|\frac{1}{m} \log \left\|\rho\left(\ell_{m}(\gamma) a\right)\right\|-\chi_{\mu}\right|<$ $\varepsilon$ and estimate the probability that $\delta\left(\rho\left(\ell_{n}(\gamma) a\right)\right)<e^{-\frac{\varepsilon}{4} n}$ among the $\gamma_{m+1}, \ldots, \gamma_{n}$ satisfying $\log \left\|\rho\left(\gamma_{n} \ldots \gamma_{m+1}\right)\right\|<2 \chi_{\mu}(n-m) \sim 2 \chi n \varepsilon$. Put $h=\rho\left(\ell_{m}(\gamma) a\right)$ and consider the balls $A(h)$ and $R(h)$. If we let

$$
\widetilde{A}\left(\rho ( \ell _ { n } ( \gamma ) a ) = \rho ( \gamma _ { n } \cdots \gamma _ { m + 1 } ) A ( h ) \text { and } \widetilde { R } \left(\rho\left(\ell_{n}(\gamma) a\right)=R(h)\right.\right.
$$

then by definition we have that

$$
\rho\left(\ell_{n}(\gamma) a\right)\left(\widetilde{R}\left(\rho\left(\ell_{n}(\gamma) a\right)^{c}\right)=\widetilde{A}\left(\rho\left(\ell_{n}(\gamma) a\right)\right.\right.
$$

and the diameters of $\widetilde{A}\left(\rho\left(\ell_{n}(\gamma) a\right)\right.$ and $\widetilde{R}\left(\rho\left(\ell_{n}(\gamma) a\right)\right.$ are not greater than $e^{-\left(\chi_{\mu}+O(\varepsilon)\right) n}$. So if we can show that these balls are separated by a distance $\geq e^{-\frac{\varepsilon}{4} n}$, we then conclude that $\rho\left(\ell_{n}(\gamma) a\right)$ is loxodromic with a fixed point in each ball, so the distance between its fixed points is $\geq e^{-\frac{\varepsilon}{4} n}$ and we are done. But if this does not happen, this means that $\rho\left(\gamma_{n} \cdots \gamma_{m+1}\right)$ maps the ball $A(h)$ very close (i.e. at distance $\leq e^{-\frac{\varepsilon}{4} n}$ ) to the ball $R(h)$. Since $h$ is fixed here, the probability of this event is governed by the properties of the unique stationary measure on $\mathbb{P}^{1}$, relative to the action of $(\rho(\Gamma), \mu)$. It follows that this probability is of order $e^{-\theta \varepsilon n}$ for some $\theta=\theta(\rho)>0$ (see [DD1, Lemma A.3] for details) and the desired result follows. 
So far we have shown that there exists $c_{1}>0$ (which we may assume is smaller than $\frac{1}{4}$ ) such that if $a$ is a fixed element in $\Gamma$ such that $\|a\| \leq e^{\frac{\varepsilon}{4} n}$, then

$$
\mathbb{P}\left(\frac{1}{n} \log \left|\operatorname{tr} \rho\left(\ell_{n}(\gamma) a\right)\right| \notin\left[\chi_{\mu}-\varepsilon, \chi_{\mu}+\varepsilon\right]\right) \leq e^{-c_{1} \varepsilon n} .
$$

Since the number of elements of norm not greater than $e^{c n}$ is equivalent to $e^{c n}$, we get that for $c_{2} \leq c_{1}$,

$$
\mathbb{P}\left(\exists a \in \Gamma \text {, s.t. }\|a\| \leq e^{c_{2} \varepsilon n}, \text { and }\left|\frac{1}{n} \log \right| \operatorname{tr} \rho\left(\ell_{n}(\gamma) a\right)\left|-\chi_{\mu}\right|>\varepsilon\right) \leq e^{\left(c_{2}-c_{1}\right) n} .
$$

By choosing $c_{2}=\frac{c_{1}}{2}$, we conclude that (17) holds with $c=\frac{c_{1}}{2}$.

Proof of Theorem 3.2. Let us first assume that $\Gamma$ is torsion-free. From now on $\mu$ is the discretization measure for the Brownian motion on $X$, as defined in $\S 2.2$. We let $S=S_{R}$ be the circle centered at 0 and of radius $R$ and work in the probability space of paths issued from $S$ in $\mathbb{H}$, endowed with the probability measure $W_{S}=\int_{S} W_{x} d s(x)$. For a generic such Brownian path, we let $T=T(\omega)$ be the stopping time defining $\mu, T_{n}=T_{n}(\omega)$ be the sequence of times obtained by repeating the stopping process, and $\tau$ be the average stopping time, which is the almost sure limit $\tau=\lim \frac{T_{n}}{n}$ (see the proof of Theorem 2.7). Observe that it is enough to prove the theorem for the sequence of radii $r_{n}=n \tau$.

The first step is the following large deviations estimate, which will be proven afterwards.

Lemma 3.4. Let $T_{n}(\omega)$ be as above. Then for large enough $n$ we have that

$$
W_{S}\left(\left\{\omega,\left|\frac{T_{n}(\omega)}{n}-\tau\right|>\varepsilon\right\}\right) \leq e^{-c(\varepsilon) n}
$$

For fixed $n$, we now define a good set $G_{n}$ of paths starting from $S$ by specifying its complement $B_{n}$. Declare that the elements $\gamma \in \Gamma$ appearing in (17) are bad. From the discretization procedure, this corresponds to a set of paths of exponentially small measure, relative to $W_{S}$, which we denote by $B_{n}^{0}$. Now by definition $\omega$ belongs to $B_{n}^{1}$ if $\left|T_{n}(\omega)-n \tau\right|>\frac{c \tau}{2 K} \varepsilon$ where $c$ is as in Proposition 3.3 and $K$ is as in Lemma 2.11. By Lemma 2.11 and the strong Markov property if $\omega \notin B_{n}^{1}$, the probability that $d_{\mathbb{H}}\left(\omega\left(T_{n}\right), \omega(n \tau)\right)$ is larger than $\frac{c \tau}{2} n \varepsilon$ is exponentially small. We let $B_{n}^{2}$ be the corresponding set of paths. Finally we put $B_{n}=B_{n}^{0} \cup B_{n}^{1} \cup B_{n}^{2}$, and $G_{n}=B_{n}^{c}$. Observe that $W_{S}\left(B_{n}\right) \leq e^{-c(\varepsilon) n}$.

We claim that if $\omega$ is good, that is $\omega \in G_{n}$, then for every $\gamma \in \Gamma \cap B_{\mathbb{H}}\left(\omega(\tau n), \frac{c \tau}{2} n \varepsilon\right)$ we have that

$$
\left|\frac{1}{n} \log \right| \operatorname{tr}(\rho(\gamma))\left|-\chi_{\mu}(\rho)\right|<\tau \varepsilon \text {, i.e. }\left|\frac{1}{2 \tau n} \log \right| \operatorname{tr}^{2}(\rho(\gamma))\left|-\chi_{\operatorname{Brown}}(\rho)\right|<\varepsilon
$$

(recall from the proof of Theorem 2.7 that $\chi_{\mu}(\rho)=\tau \chi_{\text {Brown }}(\rho)$ ). Indeed by Proposition 3.3, if $\omega \notin B_{n}$, then the element $\alpha \in \Gamma$ corresponding to $\omega\left(T_{n}\right)$ (that is, such that $\omega\left(T_{n}\right) \in \alpha(S)$ ) has the property that for every $\beta \in B_{\Gamma}(\alpha, c \tau n \varepsilon),\left|\frac{1}{n} \log \right| \operatorname{tr}(\rho(\beta))\left|-\chi_{\mu}(\rho)\right|<\tau \varepsilon$. Now, if $\omega \in G_{n}$, $\omega(\tau n)$ lies in $B_{\Gamma}\left(\alpha, \frac{c \tau}{2} n \varepsilon\right)$, whence the result.

Let $E$ be the set of group elements $\gamma \in B_{\Gamma}\left(\frac{n \tau}{2}\right)$ such that

$$
\left|\frac{1}{2 \tau n} \log \right| \operatorname{tr}^{2}(\rho(\gamma))\left|-\chi_{\operatorname{Brown}}(\rho)\right| \geq \varepsilon .
$$


To complete the proof of the theorem, we have to show that $\# E \leq e^{-c(\varepsilon) n} \# B_{\Gamma}(n \tau)$. For this, let $\widetilde{G}_{n}$ be the image of $G_{n}$ under Brownian motion at time $n \tau$, that is $\widetilde{G}_{n}=\left\{\omega(n \tau), \omega \in G_{n}\right\}$. The next lemma follows from classical estimates for the heat kernel on $\mathbb{H}$ (see below for the proof).

Lemma 3.5. The hyperbolic area of $\left(\widetilde{G}_{n}\right)^{c} \cap B_{\mathbb{H}}(0, n \tau)$ is exponentially small with respect to the area of $B_{\mathbb{H}}(0, n \tau)$, that is, there exists $c(\varepsilon)>0$ such that for large $n$,

$$
\text { Area }\left(\left(\widetilde{G}_{n}\right)^{c} \cap B_{\mathbb{H}}(0, n \tau)\right) \leq \exp (n \tau-c(\varepsilon) n) \text {. }
$$

We have shown above that if $\gamma \in E$, then $B_{\mathbb{H}}\left(\gamma, \frac{c \tau \varepsilon}{2} n\right)$ is disjoint from $\widetilde{G_{n}}$. Let $\delta=\frac{c(\varepsilon)}{2}$, where $c(\varepsilon)$ is as in the previous lemma. By the Vitali covering lemma, there exists a covering of $E$ by a finite collection of balls $\left\{B_{\mathbb{H}}\left(\gamma_{i}, \delta n\right), i=1, \ldots N\right\}$ such that the balls $B_{\mathbb{H}}\left(\gamma_{i}, \frac{\delta}{5} n\right)$ are disjoint. Thus we infer that

$$
\sum_{i=1}^{N} \operatorname{Area}\left(B_{\mathbb{H}}\left(\gamma_{i}, \frac{\delta}{5} n\right)\right)=4 \pi N \sinh ^{2}\left(\frac{\delta}{10} n\right) \leq \operatorname{Area}\left(\left(\widetilde{G}_{n}\right)^{c}\right) .
$$

Now, a well known theorem due to Margulis $[\mathrm{M}]$ (see also [EM]) asserts that when $r \rightarrow \infty$,

$$
\# B_{\Gamma}(r) \sim \frac{\operatorname{Area}\left(B_{\mathbb{H}}(0, r)\right)}{\operatorname{Area}(X)} \sim \frac{e^{r}}{\operatorname{Area}(X)} .
$$

It follows that there is a constant $C$ such that for every $r, \# B_{\Gamma}(r) \leq C e^{r}$, hence by (18) we conclude that

$$
\# E \lesssim N e^{\delta n} \lesssim \frac{e^{n \tau-c(\varepsilon) n}}{\sinh ^{2}\left(\frac{\delta}{10} n\right)} e^{\delta n} \lesssim e^{\tau n-\left(c(\varepsilon)-\frac{4}{5} \delta\right) n} \leq C e^{\tau n-\frac{c(\varepsilon)}{2} n},
$$

and the proof of the theorem is complete in the torsion free case.

Proof of Lemma 3.4. The almost sure convergence $\frac{T_{n}}{n} \rightarrow \tau$ was established in the proof of Theorem 2.7, the point here is to make this convergence more precise. As we did there we slightly shift our point of view and see our Brownian paths as sitting on $X$. We want to formalize the idea of a Brownian path issued from $S$ as obtained from the concatenation of path segments starting and ending on $S$. For this we let $\Omega_{S}$ be the set of continuous paths $[0, \infty) \rightarrow X$ starting on $S$, endowed as usual with the probability measure $W_{S}$. We define a Markov chain with values in $\Omega_{S}$ as follows: the initial distribution is $W_{S}$, and for a.e. $\omega$ the transition kernel is given by $p(\omega, \cdot)=W_{\omega(T(\omega))}$, where $T(\omega)$ is our stopping time. This Markov chain yields a sequence of random paths $\left(\omega_{n}\right)$ in $\Omega_{S}$. By the strong Markov property of Brownian motion, the law of the random path obtained by concatenating the path segments $\left.\omega_{n}\right|_{\left[0, T\left(\omega_{n}\right)\right]}$ is $W_{S}$. Associated to the Markov chain there is a real random variable $T\left(\omega_{n}\right)$ and we see that the law of $T_{n}(\omega)$ is the same as that of $\sum_{k=1}^{n} T\left(\omega_{k}\right)$.

It is easy to see that the random variables $\left(\omega_{n}\right)$ have the same law $W_{S}$. However they are not independent since $\omega_{n+1}$ must start at $\omega_{n}\left(T\left(\omega_{n}\right)\right)$. The main observation is that independence holds after two iterations. Indeed for every $x \in S$,

$$
p\left(W_{x}, \cdot\right)=\int p(\alpha, \cdot) W_{x}(d \alpha)=\int W_{\alpha(T(\alpha))} W_{x}(d \alpha)=\int W_{y} d s(y)=W_{S}
$$


because by construction the law of $\alpha(T(\alpha))$ is the circle measure $d s$. Thus we infer that for a.e. $\omega \in S$,

$$
p^{2}(\omega, \cdot)=\int p(\alpha, \cdot) p(\omega, d \alpha)=\int p(\alpha, \cdot) W_{\omega(T(\omega))}(d \alpha)=W_{S} .
$$

It follows that the random variables $\omega_{1}, \omega_{3}, \omega_{5}, \ldots$ (resp. $\left.\omega_{2}, \omega_{4}, \cdots\right)$ are independent. Furthermore, by Proposition 2.15 , there exists $s>0$ such that $\mathbb{E}\left(e^{s T(\omega)}\right)<\infty$. Therefore the desired estimate follows from the classical large deviation theorem for partial sums of i.i.d. random variables (applied separately to $\sum_{\text {even } k} T\left(\omega_{k}\right)$ and $\sum_{\text {odd } k} T\left(\omega_{k}\right)$ ).

Proof of Lemma 3.5. Let $\nu_{t}\left(\right.$ resp. $\left.\nu_{t}^{0}\right)$ be the law at time $t$ of Brownian motion with initial distribution $d s$ (resp. $\delta_{0}$ ). For notational ease, we put $F=\widetilde{G}_{n}^{c} \cap B_{\mathbb{H}}(0, n \tau)$. We know that $\nu_{n \tau}(F) \leq W_{S}\left(B_{n}\right) \leq e^{-c(\varepsilon) n}$, and from this we want to derive an estimate for the hyperbolic area of $F$. Let $\delta>0$ to be specified later, and decompose $F$ as

$$
F=\left(F \cap B_{\mathbb{H}}(0, n \tau-n \delta)\right) \cup\left(F \cap A_{\mathbb{H}}(n \tau-n \delta, n \tau)\right)
$$

where $A_{\mathbb{H}}(r, s)$ denotes the annular region centered at 0 with inner radius $r$ and outer radius $s$. The first observation is that

$$
\text { Area }\left(F \cap B_{\mathbb{H}}(0, n \tau-n \delta)\right) \leq \operatorname{Area}\left(B_{\mathbb{H}}(0, n \tau-n \delta)\right) \lesssim \exp (n \tau-n \delta) .
$$

The probability measure $\nu_{t}^{0}$ is radial, and from the estimate for its radial density $k^{0}(t, r)$ given in (4), we deduce that for $t=n \tau$ and $n \tau-n \delta \leq r \leq n \tau$,

$$
\frac{1}{\sqrt{n}} e^{-n \frac{\delta^{2}}{4 \tau}} \lesssim k^{0}(n \tau, r) \lesssim \frac{1}{\sqrt{n}}
$$

Since $\nu_{n \tau}$ is an average of bounded translates of $\nu_{n \tau}^{0}$, the same estimate holds for the radial density $k(n \tau, r)$ of $\nu_{n \tau}$. On the other hand the radial density of hyperbolic area is $r \sinh r$, so we infer that

$$
\begin{aligned}
\operatorname{Area}\left(F \cap A_{\mathbb{H}}(n \tau-n \delta, n \tau)\right) & \leq \int_{F \cap A_{\mathbb{H}}(n \tau-n \delta, n \tau)} r(\sinh r) d r d \theta \\
& \lesssim \int_{F \cap A_{\mathbb{H}}(n \tau-n \delta, n \tau)} r(\sinh r) k(n \tau, r) \sqrt{n} e^{n \frac{\delta^{2}}{4 \tau}} d r d \theta \\
& \lesssim n^{3 / 2} \exp \left(n \tau+\frac{\delta^{2}}{4 \tau} n\right) \nu_{n \tau}(F) \leq n^{3 / 2} \exp \left(n \tau+\frac{\delta^{2}}{4 \tau} n-c(\varepsilon) n\right) .
\end{aligned}
$$

Finally we get that

$$
\operatorname{Area}(F) \lesssim \exp (n \tau-n \delta)+n^{3 / 2} \exp \left(n \tau+\frac{\delta^{2}}{4 \tau} n-c(\varepsilon) n\right),
$$

so by choosing $\delta=\sqrt{\tau c(\varepsilon)}$ we are done.

Let us now assume that $\Gamma$ has torsion, and briefly explain how to adapt the proof of the theorem. We pick a torsion-free subgroup $\Gamma^{\prime} \leq \Gamma$ of finite index, and apply the first part of the proof to $\Gamma^{\prime}$. In this way we get a discretization measure $\mu$ on $\Gamma^{\prime}$. We slightly modify Proposition 3.3 to allow for a perturbation $a$ belonging to $\Gamma$ (this only slightly affects the counting in the last part of the proof), so that its conclusion now reads

$$
\mu^{n}\left(\left\{\gamma \in \Gamma^{\prime}, \exists a \in \Gamma \text { s.t. }\|a\| \leq e^{c \varepsilon n} \text { and }\left|\frac{1}{n} \log \right| \operatorname{tr} \rho(\gamma a)\left|-\chi_{\mu}\right|>\varepsilon\right\}\right) \leq \exp (-c \varepsilon n) .
$$


Then we argue exactly as in the torsion free case, by introducing the set $E$ of $\gamma \in \Gamma \cap B_{\mathbb{H}}(n \tau)$ such that $\left|\frac{1}{2 \tau n} \log \right| \operatorname{tr}^{2}(\rho(\gamma))\left|-\chi_{\text {Brown }}(\rho)\right| \geq \varepsilon$. To the discretized Brownian motion on $\Gamma^{\prime}$, we associate the sets $G_{n}$ and $\widetilde{G}_{n}$ exactly as before, and we estimate \#E by arguing that, from the modified version of Proposition 3.3, if $\gamma \in E$, then $B_{\mathbb{H}}\left(\gamma, \frac{c \tau \varepsilon}{2} n\right)$ is disjoint from $\widetilde{G_{n}}$. This completes the proof of the theorem. 3.1 .

The following corollary, of independent interest, is the key step in the proof of Theorem

Corollary 3.6. Let $\Gamma$ be a lattice in $\operatorname{PSL}(2, \mathbb{R})$, and $\rho$ be a non-elementary parabolic representation of $\Gamma$. If $\left(\gamma_{n}\right)$ is an admissible random sequence in $\Gamma$, then almost surely,

$$
\frac{1}{2 d\left(\gamma_{n}\right)} \log \left|\operatorname{tr}^{2}\left(\rho\left(\gamma_{n}\right)\right)\right| \underset{n \rightarrow \infty}{\longrightarrow} \chi_{\text {Brown }}(\rho) .
$$

Proof. Recall that admissible means that the $\gamma_{n}$ are chosen randomly in $B_{\Gamma}\left(r_{n}\right)$, relative to the counting measure, where $r_{n}$ is a sequence such that $\sum e^{-c r_{n}}$ converges for every $c>0$.

Fix $\varepsilon>0$. We will show that

$$
\frac{1}{\# B_{\Gamma}\left(r_{n}\right)} \#\left\{\gamma \in B_{\Gamma}\left(r_{n}\right),\left|\frac{1}{2 d\left(\gamma_{n}\right)} \log \right| \operatorname{tr}^{2}\left(\rho\left(\gamma_{n}\right)\right)\left|-\chi_{\operatorname{Brown}}(\rho)\right|>\varepsilon\right\} \lesssim e^{-c(\varepsilon) r_{n}} .
$$

We then deduce from the Borel-Cantelli lemma that a.s. for large $n, \frac{1}{2 d\left(\gamma_{n}\right)} \log \left|\operatorname{tr}^{2}\left(\rho\left(\gamma_{n}\right)\right)\right|$ is $\varepsilon$-close to $\chi_{\operatorname{Brown}}(\rho)<\varepsilon$, and the result follows.

The first observation is that in $B_{\Gamma}\left(r_{n}\right)$, by the Margulis estimate (19) on \# $B_{\Gamma}(r)$, for every $\delta>0$, we have that

$$
\#\left\{\gamma, d\left(\gamma_{n}\right)<(1-\delta) r_{n}\right\} \lesssim e^{(1-\delta) r_{n}} .
$$

We choose $\delta=\min \left(\frac{1}{2}, \frac{\varepsilon}{4 \chi}\right)$. Now if

$$
d\left(\gamma_{n}\right) \geq(1-\delta) r_{n} \text { and }\left|\frac{1}{2 d\left(\gamma_{n}\right)} \log \right| \operatorname{tr}^{2}\left(\rho\left(\gamma_{n}\right)\right)\left|-\chi_{\operatorname{Brown}}(\rho)\right|>\varepsilon,
$$

an elementary computation shows that

$$
\left|\frac{1}{2 r_{n}} \log \right| \operatorname{tr}^{2}\left(\rho\left(\gamma_{n}\right)\right)\left|-\chi_{\operatorname{Brown}}(\rho)\right|>(1-\delta) \varepsilon-\delta \chi_{\operatorname{Brown}}(\rho)>\frac{\varepsilon}{4}
$$

Therefore the desired estimate (20) follows from (21), Theorem 3.2 and the fact that \# $B_{\Gamma}\left(r_{n}\right)$ grows like $e^{r_{n}}$.

Remark 3.7. This corollary yields yet another definition of the natural Lyapunov exponent, where the choice of Riemann surface structure is reflected in the fact of counting in the lattice $\Gamma$. Similarly, we could replace the trace by the norm, and assert that generically, $\frac{1}{2 d\left(\gamma_{n}\right)} \log \left\|\rho\left(\gamma_{n}\right)\right\|$ converges to $\chi_{\text {Brown }}(\rho)$. The proof is identical to that of Theorem 3.2 (observe that Proposition 3.3 becomes much simpler in this case) .

3.2. Proof of Theorem 3.1. Let $m_{n}$ be the normalized counting measure on $B_{\Gamma}\left(r_{n}\right)$ and $m=\prod_{n \geq 1} m_{n}$. By considering the potentials of the currents $\frac{1}{2 d\left(\gamma_{n}\right)}\left[Z\left(\gamma_{n}, t\right)\right]$, it is enough to show that for $m$-a.e. sequence $\gamma=\left(\gamma_{n}\right)$ in $\Gamma^{\mathbb{N}}$, the sequence of psh functions $(\lambda \mapsto$ $u(\lambda, \gamma, n))_{n \geq 1}$ on $\Lambda$ defined by

$$
u(\lambda, \gamma, n)=\frac{1}{2 d\left(\gamma_{n}\right)} \log \left|\operatorname{tr}^{2}\left(\rho_{\lambda}\left(\gamma_{n}\right)\right)-t\right|
$$


converges in $L_{\text {loc }}^{1}(\Lambda)$ to $\lambda \mapsto \chi_{\text {Brown }}\left(\rho_{\lambda}\right)$. Corollary 3.6 above implies that for every fixed parameter $\lambda, u(\lambda, \gamma, n)$ converges $m$-almost surely to $\chi_{\operatorname{Brown}}\left(\rho_{\lambda}\right)$. Now clearly the set $E \subset$ $\Lambda \times \Gamma^{\mathbb{N}}$ of parameters $(\lambda, \gamma)$ such that $u(\lambda, \gamma, n)$ converges to $\chi_{\operatorname{Brown}}\left(\rho_{\lambda}\right)$ is measurable, so by Fubini's theorem it has full $d \lambda \otimes m$ measure. Reverting the order of integration (Fubini's theorem again), we infer that for $m$ a.e. $\gamma, u(\lambda, \gamma, n)$ converges to $\chi_{\text {Brown }}\left(\rho_{\lambda}\right) d \lambda$-a.s.

In addition, the sequence $u(\lambda, \gamma, n)$ is locally uniformly bounded from above on $\Lambda$. Indeed we have that

$$
\begin{aligned}
\frac{1}{2 d\left(\gamma_{n}\right)} \log \left|\operatorname{tr}^{2}\left(\rho_{\lambda}\left(\gamma_{n}\right)\right)-t\right| & \left.\leq \frac{1}{2 d\left(\gamma_{n}\right)} \log \| \rho_{\lambda}\left(\gamma_{n}\right)\right) \|^{2}+O(1) \\
& \leq \frac{\beta_{\rho_{\lambda}}}{d\left(\gamma_{n}\right)} \log \left\|\gamma_{n}\right\|+O(1) \leq \frac{\beta_{\rho_{\lambda}}}{2}+O(1)
\end{aligned}
$$

where the constant $\beta_{\rho_{\lambda}}$ is locally uniform in $\Lambda$ (see Corollary 2.3). By the standard compactness theorems for sequences of subharmonic functions (see Theorems 3.2.12, 3.2.13 and 3.4.14 in [Hö]), we conclude that $u(\lambda, \gamma, n)$ converges to $\chi_{\operatorname{Brown}}\left(\rho_{\lambda}\right)$ in $L_{\text {loc }}^{1}(\Lambda)$. Indeed, if $\gamma$ is such that convergence holds a.e in $\Lambda$, we can extract a subsequence converging to some $v$ in $L_{\text {loc }}^{1}$, and $\lim \sup _{n \rightarrow \infty} u(\lambda, \gamma, n)=v$ outside a polar set, therefore $v=\chi_{\text {Brown }}$. This finishes the proof.

Remark 3.8. As for Theorem 3.2, the statement of Theorem 3.1 may be adapted to the case where $\Gamma$ admits non-trivial torsion.

3.3. Random closed geodesics. Let $\mathrm{CG}(X)$ be the set of closed geodesics in $X$, which is a countable set. We allow a geodesic to be travelled several times. If this does not happen, the geodesic is said to be primitive.

Let $\rho \in \operatorname{Hom}_{\text {par }}(G, \operatorname{PSL}(2, \mathbb{C}))$. If $\gamma \in \operatorname{CG}(X)$ the holonomy $h_{\rho}(\gamma)$ is defined up to conjugacy in $\rho(\Gamma)$, so it makes sense to talk about its (squared) $\operatorname{trace}^{2} \operatorname{tr}^{2} h_{\rho}(\gamma)$. If now $\left(\rho_{\lambda}\right)$ is family of parabolic representations, for $t \in \mathbb{C}$ we may consider the subvariety in $\Lambda$ defined by

$$
Z(\gamma, t)=\left\{\lambda \in \Lambda, \operatorname{tr}^{2} h_{\rho_{\lambda}}(\gamma)=t\right\} .
$$

Here we first briefly review several notions of "random closed geodesics" on $X$, and interpret Theorem 3.1 in these terms.

3.3.1. Thurston model. To start with, assume that $X$ is compact. The following model for random geodesics on $X$ was apparently popularized by Thurston (see e.g. [Bo]): for $(x, v) \in$ $S X$ let as before $\gamma_{(x, v)}$ be the associated unit speed geodesic ray. For $t>0$, close the path $\left.\gamma_{(x, v)}\right|_{[0, t]}$ with a shortest path returning to $x$. Notice that there may be a choice involved here, which we may disregard for it happens only for a set of $(x, v)$ of zero measure. We denote by $\operatorname{cg}(x, v)(t)$ the unique closed geodesic freely homotopic to the obtained loop. Notice that its length may be much smaller than $t$. Nevertheless it follows from Theorem 3.2 applied to the identity representation that this phenomenon happens only on a set of exponentially small measure.

In this way we have constructed a family of projections $v \mapsto \operatorname{cg}(x, v)(t)$ from $S X$ to $\operatorname{CG}(X)$ indexed by $t$. Projecting the (normalized) Liouville measure gives rise to a family of probability measures $\left(m_{t}^{\text {geodesic }}\right)_{t>0}$ on $\mathrm{CG}(X)$.

Several variants of this construction may be considered:

- Variant 1: a first possibility is to restrict to geodesic paths starting from the marked point $\star$, and close them so as to obtain loops based at $\star$. 
- Variant 2: allow for paths starting from any $(x, v)$, but append them at the two endpoints with shortest paths joining them to $\star$.

Notice that in these two variants, we naturally obtain a family of measures on the fundamental group, which in turn projects to a family of measures on $\operatorname{CG}(X)$. Notice also that in the second variant, the family of measures that we obtain in $\operatorname{CG}(X)$ is the same as in the original Thurston construction (since the corresponding loops are conjugate in $\Gamma$ ). In any case, the measures $\left(m_{t}^{\text {geodesic }}\right)_{t>0}$ may be thought of as measures on $\Gamma$. A good intuitive picture for $m_{t}^{\text {geodesic }}$ is that of being loosely equidistributed on an annulus of bounded width about the circle of radius $t$ in $\Gamma \subset \mathbb{H}$.

These variants are interesting because they also make sense when $X$ is only of finite volume. Indeed, using these constructions we obtain a family of measures $\left(m_{t}^{\text {geodesic }}\right)_{t>0}$ on $G$. Now $\gamma \in \Gamma$ projects onto a closed geodesic if and only if it is not parabolic. Again by Theorem 3.2 this happens outside a set of exponentially small measure. So what we do is simply to restrict to loxodromic elements, renormalize the mass and project to $\mathrm{CG}(X)$ we also obtain in this way a reasonable model for random closed geodesics on $X$.

The following lemma asserts that the measures $\left(m_{t}^{\text {geodesic }}\right)$ are well spread in $\Gamma$.

Lemma 3.9. Let $X$ be a hyperbolic Riemann surface of finite type endowed with its hyperbolic metric, and let $\left(m_{t}^{\text {geodesic }}\right)_{t>0}$ be any of the family of measures on $\Gamma$ constructed above. Then for every $\delta>0$ there exist constants $K$ and $c$ such that for all $t>0$, for every subset $A \subset \Gamma$ with cardinality $\# A \leq e^{(1-\delta) t}$, we have that $m_{t}^{\text {geodesic }}(A) \leq K e^{-c t}$.

Proof. Let us treat the case of paths starting from $\star$ (Variant 1 above). If $v$ is a unit tangent vector at $\star$, we denote by $\gamma_{v}$ the unit speed geodesic ray starting from $\star$ in the direction of $v$.

The first claim is that there exists a constant $K_{1}$ depending only on $X$ such that the probability that $d_{X}\left(\gamma_{v}(t), \star\right)$ is larger than $\frac{\delta}{2} t$ is not greater than $K_{1} e^{-\delta t / 2}$. A proof of this fact is outlined in [Su1, §9], and discussed with much greater detail and generality in [KM].

For $t>0$, let $E_{t}$ be the set of tangent vectors $v \in T_{\star}^{1} X$ such that $d_{X}\left(\gamma_{v}(t), \star\right) \leq \frac{\delta}{2} t$, which by our previous claim has measure $\geq 1-K_{1} e^{\delta t / 2}$. We lift the situation to $\mathbb{H}$, so that our geodesics are now rays of hyperbolic length $t$ issued from 0 . If a lattice point $\gamma \in \Gamma \cdot 0$ is the endpoint of $\widetilde{\gamma}_{v}(t)$ for some $v \in E_{t}$, then $d_{\mathbb{H}}\left(\gamma, \gamma_{v}(t)\right) \leq \frac{\delta}{2} t$. We apply the first cosine rule [Bea, $\S 7.12]$ in the triangle $T\left(0, \gamma, \widetilde{\gamma}_{v}(t)\right)$ to get an estimate of the angle $\theta$ at the origin between the rays $\gamma_{v}(t)$ and $[0, \gamma]$. It follows that $\cos (\theta)=1-C e^{-(2+\alpha) t}+O\left(e^{-3 t}\right)$, with $|\alpha| \leq \delta$, from which we deduce that $\theta \leq K_{2} e^{-(1-\delta / 2) t}$. Hence for any subset $A$ of $\Gamma$, the set of $v \in E_{t}$ such that $\widetilde{\gamma}_{v}(t)$ belongs to $A$ has measure at most $K_{2}(\# A) e^{-(1-\delta / 2) t}$.

We conclude that if $A$ is as in the statement of the lemma, the measure of the set of tangent vectors $v$ such that $\widetilde{\gamma}_{v}(t)$ belongs to $A$ is not greater than $\left(K_{1}+K_{2}\right) e^{-\frac{\delta}{2} t}$ and the result follows.

It is an entertaining exercise in hyperbolic geometry to show that the cardinality of the set of $\gamma \in B_{\Gamma}(t)$ which are proper powers of elements of $\Gamma$ is of order of magnitude $e^{t / 2}$. It follows that a generic geodesic with respect to the measure $m_{t}^{\text {geodesic }}$ is primitive.

3.3.2. Brownian Thurston model. We can do exactly the same construction by using Brownian paths instead of geodesic ones. As before, several variants are possible, by choosing the initial point to be either fixed or distributed according to the Poincaré volume element on $X$, and by choosing the paths to be closed to become a loop based at $\star$ or not. In this way we 
obtain families of measures on $\Gamma$ or on $\operatorname{CG}(X)$, which, abusing slightly, will be given the same notation $\left(m_{t}^{\text {Brownian }}\right)_{t>0}$. In the unit disk picture, the corresponding measures on $\Gamma$ are induced by the heat flow on $\mathbb{H}^{2}$, so they now look like Gaussian measures concentrated around the circle of radius $t$.

Again, these measures are well spread on $\Gamma$.

Lemma 3.10. Let $X$ be as above and $\left(m_{t}^{\text {Brownian }}\right)_{t>0}$ be the family of measures on $\Gamma$ obtained from the Brownian model for random geodesics. Then for every $\delta>0$ there exist constants $K$ and $c$ such that for all $t>0$, for every subset $A \subset \Gamma$ with cardinality $\# A \leq e^{(1-\delta) t}$, it holds that $m_{t}^{\text {Brownian }}(A) \leq K e^{-c t}$.

The proof uses arguments similar to those of Theorem 3.2, and will only be sketched. Details will be left as an exercise to the reader.

We need the following estimate.

Lemma 3.11. Let $(X, \star)$ be as above. There exists a positive constant $c$ such that

$$
\mathbb{P}_{\star}\left(d_{X}(\star, \omega(t)) \geq c t\right) \lesssim e^{-c t / 2} .
$$

Proof (sketch). Let $f$ be the density of the diffusion of $\delta_{\star}$ at time 1 , relative to the hyperbolic area element on $X$. The classical Li-Yau estimates on the heat kernel (see e.g. [CC, §B.7]) imply that $f \in L^{\infty}(X) \subset L^{2}(X)$. Let $\left(\Pi_{t}\right)_{t>0}$ be the Brownian semigroup on $X$, and put $V_{t}=\left\{x \in X, d_{X}(x, \star) \geq c t\right\}$. We have to show that $\int_{V_{t}} \Pi_{t} f$ is exponentially small with $t$. For this, using the Schwarz inequality we write

$$
\left|\int_{V_{t}} \Pi_{t} f\right| \leq\left\|\Pi_{t} f\right\|_{L^{2}(X)} \operatorname{vol}\left(V_{t}\right)^{1 / 2}
$$

and we conclude using the fact that $\left\|\Pi_{t} f\right\|_{L^{2}(X)}$ converges, while vol $\left(V_{t}\right)$ decreases exponentially with $t$.

Proof of Lemma 3.10 (sketch). We lift the situation to $\mathbb{H}$, and look at Brownian paths issued from 0. Fix a constant $c>0$. By the estimates (4) for the heat kernel on $\mathbb{H}$ and the above lemma, the probability that $\omega(t) \notin A_{\mathbb{H}}(t(1-c), t(1+c))$ or $d_{\mathbb{H}}(\omega(t), \Gamma) \geq c t$ decreases exponentially with $t$. We discard this set of paths, so that it is enough to work with $A^{\prime}=$ $A \cap A_{\mathbb{H}}(t(1-3 c), t(1+3 c))$ instead of $A$. Now if we denote by $\widetilde{\omega}_{t}$ the concatenation of $\left.\omega\right|_{[0, t]}$ with a shortest path joining $\omega(t)$ to $\Gamma$, we infer that $\mathbb{P}\left(\widetilde{\omega}_{t} \in A^{\prime}\right) \leq \nu_{t}\left(\bigcup_{a \in A^{\prime}} B(a, c t)\right)$, where $\nu_{t}$ is the law of Brownian motion at time $t$. We compare this probability to the hyperbolic area of $\bigcup_{a \in A} B(a, c t)$ by using (4) again, and get an estimate of the form $\mathbb{P}\left(\widetilde{\omega}_{t} \in A^{\prime}\right) \lesssim$ $\exp ((-\delta+5 c) t)$. Finally, choosing the constant $c$ small enough with respect to $\delta$ finishes the proof.

3.3.3. Length-based model. Let $\mathrm{CG}_{t}(X)$ be the set of primitive geodesics of length at most $t$ on $X$, and let $m_{t}^{\text {length }}$ be simply the normalized counting measure on $\mathrm{CG}_{t}(X)$.

To apply Theorem 3.1 we need to to lift $m_{t}^{\text {length }}$ to $\Gamma$. For this, we choose a shortest path from $\star$ to a given geodesic to make it a loop based at $\star$. Observe that the length of this path is uniformly bounded. Indeed, recall from Proposition 2.2 that there are neighborhoods $C_{i}$ of the cusps such that each geodesic must enter the compact region $X \backslash \bigcup C_{i}$.

We then infer that the lifted measure is supported in an annulus of bounded width about the circle of radius $t$ in $\Gamma$. Also, the lifted measure is well spread in $\Gamma$ in the sense of Lemmas 3.9 and 3.10 because of the well-known asymptotic estimate $\mathbb{H C G}_{t}(X) \sim \frac{e^{t}}{t}$ as $t \rightarrow \infty$. This 
estimate originates in the works of Huber and Selberg (references covering the non-compact case include [He, I])

3.3.4. Equidistribution. We now choose any of the above models for random geodesics, and denote by $\left(m_{t}\right)$ the associated family of measures on $\mathrm{CG}(X)$. Let us recall the statement of the equidistribution theorem on $\Lambda$ associated to random closed geodesics (Theorem $\mathrm{C}$ ).

Theorem 3.12. Let $X$ be a hyperbolic Riemann surface of finite type, and $\left(\rho_{\lambda}\right)_{\lambda \in \Lambda}$ be a holomorphic family of representations of $G=\pi_{1}(X)$, satisfying (R1, R2, R3). Let $T_{\text {bif }}=$ $d d^{c} \chi_{\text {Brown }}$ be the natural bifurcation current.

Let $\left(t_{n}\right)$ be a sequence of positive numbers such that for every $c>0$ the series $\sum e^{-c t_{n}}$ converges. Let $\left(\gamma_{n}\right)$ be a random sequence of closed geodesics on $X$, according to the product measure $\prod m_{t_{n}}$. Then almost surely we have that

$$
\frac{1}{2 \operatorname{length}\left(\gamma_{n}\right)}\left[Z\left(\gamma_{n}, t\right)\right] \underset{n \rightarrow \infty}{\longrightarrow} T_{\text {bif }}
$$

The proof of the theorem is identical to that of Theorem 3.1, using the fact that the lifted measures $m_{t_{n}}$ are well spread on $\Gamma$. Indeed for fixed $\lambda$, a Borel-Cantelli argument based on Theorem 3.2 together with Lemma 3.9 (Thurston model), Lemma 3.10 (Brownian Thurston model) or the estimate $\# \mathrm{CG}_{t}(X) \sim \frac{e^{t}}{t}$ (length-based model) implies that almost surely

$$
\frac{1}{2 \text { length }\left(\gamma_{n}\right)} \log \left|\operatorname{tr}^{2}\left(\rho\left(\gamma_{n}\right)\right)\right| \underset{n \rightarrow \infty}{\longrightarrow} \chi_{\text {Brown }}(\rho),
$$

which is the statement of Theorem D. Theorem 3.12 (i.e. Theorem C) then follows exactly as in $\S 3.2$.

\section{FURThER REMARKS, OPEN PROBLEMS}

4.1. A first natural problem is to study the dependence of the natural Lyapunov exponent $\chi_{\text {Brown }}$ as a function of the Riemann surface structure $X$. It is likely that this function is continuous in $(\rho, X)$. It would be interesting to study the possible subharmonicity properties of this function as $X$ varies in Teichmüller space.

4.2. (cf. [DD1, §5.2.3]). The varieties $Z(\gamma, 4)$ appearing in the equidistribution theorems are made of accidental parabolics and new relations. The question is to determine if one of the two types dominates. At first sight one might guess that the varieties of accidental relations $\left\{\lambda, \rho_{\lambda}(\gamma)=\mathrm{id}\right\}$ are of codimension 3 in the representation variety. Unfortunately this is wrong. Indeed, if $\operatorname{tr}^{2}(\rho(\gamma))=0$ (a codimension 1 condition) then $\rho\left(\gamma^{2}\right)=$ id. More generally for any $k$ we can obtain hypersurfaces of representations such that $\rho\left(\gamma^{k}\right)=$ id. On the other hand, as observed in $§ 3.3$, random geodesics are generically primitive, so this phenomenon is negligible (see $[\mathrm{Lu}]$ for a wide generalization of this fact).

Is it true that for a generic sequence $\left(\gamma_{n}\right)$ of closed geodesics, $\left\{\lambda, \rho_{\lambda}\left(\left[\gamma_{n}\right]\right)=\mathrm{id}\right\}$ is asymptotically of codimension greater than 1 in the representation variety?

4.3. Our results may be recast in the more general framework of holomorphic families of $\operatorname{PSL}(2, \mathbb{C})$-cocycles over a discrete or continuous time dynamical system, endowed with some invariant measure. A general question in this area of research is how the values of this cocycle over the set of periodic orbits approximates the Lyapunov exponent (see e.g. [Kal]). What Theorem D says is that we can have very precise such results (with exponential deviation estimates) in the particular case of the geodesic flow in constant negative curvature. It 
would be interesting to know how this can be generalized to a more general (say, uniformly hyperbolic) base dynamics. One possible way of doing this would be to use the theory of Markov driven random products of matrices, which was developed in particular by Guivarc'h and his collaborators (see [Gu] for a review).

4.4. What can be said about the distribution of $\left[Z\left(\gamma_{n}, 4\right)\right]$ when the $\gamma_{n}$ are simple closed geodesics on $X$ ?

\section{REFERENCES}

[Al] Alvarez, Sébastien. Discretization of harmonic measures for foliated bundles. C. R. Math. Acad. Sci. Paris 350 (2012), 621-626.

[An] Ancona, Alano. Théorie du potentiel sur les graphes et les variétés. École d'été de Probabilités de Saint-Flour XVIII, 1-112, Lecture Notes in Math., 1427, Springer, Berlin, 1990.

[Ao] Aoun, Richard. Random subgroups of linear groups are free. Duke Math. J. 160 (2011), 117-173.

[BaLe] Ballmann, Werner; Ledrappier, François. Discretization of positive harmonic functions on Riemannian manifolds and Martin boundary. Actes de la Table Ronde de Géométrie Différentielle (Luminy, 1992), 77-92, Sémin. Congr., 1, Soc. Math. France, Paris, 1996.

[Bea] Beardon, Alan F. The geometry of discrete groups. Graduate Texts in Mathematics, 91. SpringerVerlag, New York, 1983.

[Bo] Bonahon, Francis. The geometry of Teichmüller space via geodesic currents. Invent. Math. 92 (1988), no. 1, 139-162.

[BGM] Bonatti, Christian; Gómez-Mont, Xavier; Viana, Marcelo. Généricité d'exposants de Lyapunov nonnuls pour des produits déterministes de matrices. Ann. Inst. H. Poincaré Anal. Non Linéaire 20 (2003), 579- 624 .

[BL] Bougerol, Philippe; Lacroix, Jean. Products of random matrices with applications to Schrödinger operators. Progress in Probability and Statistics, 8. Birkhäuser Boston, Inc., Boston, MA, 1985.

[CC] Candel, Alberto; Conlon, Lawrence. Foliations. II. Graduate Studies in Mathematics, 60. American Mathematical Society, Providence, RI, 2003.

[CK] Carmona, René; Klein, Abel. Exponential moments for hitting times of uniformly ergodic Markov processes. Ann. Probab. 11 (1983), no. 3, 648-655.

[Da] Davies, Edward B. Heat kernels and spectral theory. Cambridge Tracts in Mathematics, 92. Cambridge University Press, Cambridge, 1989.

[DD1] Deroin, Bertrand; Dujardin, Romain. Random walks, Kleinian groups, and bifurcation currents. Invent. Math. 190 (2012) 57-118.

[DD2] Deroin, Bertrand; Dujardin, Romain. Complex projective structures Lyapunov exponent, degree and harmonic measure. Preprint (2013).

[DDp] Deroin, Bertrand; Dupont Christophe. Topology and dynamics of laminations in surfaces of general type. Preprint (2012), arXiv:1203.6244.

[DKN] Deroin, Bertrand; Kleptsyn, Victor; Andrés Navas. On the question of ergodicity for minimal group actions on the circle. Mosc. Math. J. 9 (2009), 263-303

[Duj] Dujardin, Romain. Bifurcation currents and equidistribution on parameter space. Frontiers in Complex Dynamics, celebrating J. Milnor's 80th birthday, to appear.

[Dum] Dumas, David. Complex projective structures. Handbook of Teichmüller theory. Vol. II, 455-508, Eur. Math. Soc., Zürich, 2009.

[EM] Eskin, Alex; McMullen, Curtis T. Mixing, counting, and equidistribution in Lie groups. Duke Math. J. 71 (1993), 181-209.

[FL] Franchi, Jacques; Le Jan, Yves. Hyperbolic dynamics and Brownian motion: an introduction. Oxford Mathematical Monographs, Oxford Univ. Press, 2012.

[F1] Furstenberg, Hillel Noncommuting random products. Trans. Amer. Math. Soc. 108 (1963), 377-428.

[F2] Furstenberg, Hillel. Random walks and discrete subgroups of Lie groups. 1971 Advances in Probability and Related Topics, Vol. 1 pp. 1-63 Dekker, New York

[Gri] Grigor'yan, Alexander. Analytic and geometric background of recurrence and non-explosion of the Brownian motion on Riemannian manifolds. Bull. Amer. Math. Soc. (N.S.) 36 (1999), 135-249. 
[Gru] Gruet, Jean-Claude. On the length of the homotopic Brownian word in the thrice punctured sphere. Probab. Theory Related Fields 111 (1998), no. 4, 489-516.

[Gu] Guivarc'h, Yves. On contraction properties for products of Markov driven random matrices. J. Math. Phys., Anal., Geom. 4 (2008), 457-489.

[GL] Guivarc'h, Yves; Le Jan, Yves. Winding of the geodesic flow on modular surfaces. Ann. Scient. Ec. Norm. 26 (1993), 23-50.

[He] Hejhal, Dennis A. The Selberg trace formula for PSL(2, R), vol. I, II. Lecture Notes in Mathematics, vol. 548 and 1001. Springer-Verlag, Berlin.

[Hö] Hörmander, Lars. Notions of convexity. Progress in Math 127. Birkhäuser, Boston, MA, 1994.

[I] Iwaniec, Henryk. Spectral methods of automorphic forms. Second edition. Graduate Studies in Mathematics, 53. American Mathematical Society, Providence, RI; Revista Matemética Iberoamericana, Madrid, 2002.

[Kai] Kaimanovich, Vadim. Discretization of bounded harmonic functions on Riemannian manifolds and entropy. Potential theory (Nagoya, 1990), 213-223, de Gruyter, Berlin, 1992.

[KV] Kaimanovich, Vadim; Vershik, Anatoly. Random walks on discrete groups: boundary and entropy. Ann. Probab. 11 (1983), no. 3, 457-490.

[Kal] Kalinin, Boris. Livšic theorem for matrix cocycles. Ann. Math. 173 (2011), 1025-1042.

[Kap] Kapovich, Michael Hyperbolic manifolds and discrete groups. Progress in Mathematics, 183. Birkhéuser Boston, Inc., Boston, MA, 2001.

[KM] Kleinbock, Dmitry; Margulis, Gregory A. Logarithm laws for flows on homogeneous spaces. Invent. math. 138 (1999), 451-494.

[Le] Le Page, Émile. Régularité du plus grand exposant caractéristique des produits de matrices aléatoires indépendantes et applications. Ann. Inst. H. Poincaré Probab. Statist. 25 (1989), no. 2, 109-142.

[Lu] Lubotzky, Alexander; Meiri, Chen. Sieve methods in group theory I: powers in linear groups. J. Am. Math. Soc. 25 (2012), 1119-1148.

[LS] Lyons, Terry; Sullivan, Dennis. Function theory, random paths and covering spaces. J. Differential Geom. 19 (1984), no. 2, 299-323.

[M] Margulis, Grigoriy A. On some aspects of the theory of Anosov systems. With a survey by Richard Sharp. Springer Monographs in Mathematics. Springer-Verlag, Berlin, 2004.

[Su1] Sullivan, Dennis Disjoint spheres, approximation by imaginary quadratic numbers, and the logarithm law for geodesics. Acta Math. 149 (1982), 215-237.

[Su2] Sullivan, Dennis. Quasiconformal homeomorphisms and dynamics. II. Structural stability implies hyperbolicity for Kleinian groups. Acta Math. 155 (1985), no. 3-4, 243-260.

CNRS, Département de Mathématique d'Orsay, Bâtiment 425, Université de Paris Sud, 91405 Orsay CEDEX, France.

E-mail address: bertrand.deroin@math.u-psud.fr

LAMA, Université Paris-Est Marne-la-Vallée, 5 Boulevard Descartes, 77454 Champs sur Marne, France

E-mail address: romain.dujardin@univ-mlv.fr 